\title{
NORMAL FORMS FOR THE ENDPOINT MAP NEAR NICE SINGULAR CURVES FOR RANK-TWO DISTRIBUTIONS*,**
}

\author{
Andrei A. Agrachev ${ }^{1}$ And Francesco Boarotto B $^{2 * * *}$
}

\begin{abstract}
Given a rank-two sub-Riemannian structure $(M, \Delta)$ and a point $x_{0} \in M$, a singular curve is a critical point of the endpoint map $F: \gamma \mapsto \gamma(1)$ defined on the space of horizontal curves starting at $x_{0}$. The typical least degenerate singular curves of these structures are called regular singular curves; they are nice if their endpoint is not conjugate along $\gamma$. The main goal of this paper is to show that locally around a nice singular curve $\gamma$, once we choose a suitable topology on the control space we can find a normal form for the endpoint map, in which $F$ writes essentially as a sum of a linear map and a quadratic form. This is a preparation for a forthcoming generalization of the Morse theory to rank-two sub-Riemannian structures.
\end{abstract}

Mathematics Subject Classification. 53C17, 58K50, 58K05.

Received July 23, 2019. Accepted November 30, 2020.

\section{INTRODUCTION}

\subsection{Horizontal path spaces and singular curves}

Let $M$ be a smooth manifold of dimension $m>2$ and consider a smooth (i.e. $C^{\infty}$-smooth), totally nonholonomic distribution $\Delta \subset T M$ of rank 2. The pair $(M, \Delta)$ is called a sub-Riemannian structure of rank two. Define $I:=[0,1]$. Given a point $x_{0} \in M$ (which we will assume fixed once and for all) the horizontal path space $\Omega$ of admissible (also called horizontal) curves starting at $x_{0}$ is defined by:

$$
\Omega=\left\{\gamma: I \rightarrow M \mid \gamma(0)=x_{0}, \gamma \text { is absolutely continuous, } \dot{\gamma} \in \Delta \text { a.e. and is } L^{2} \text {-integrable }\right\} \text {. }
$$

The $W^{1,2}$-topology endows $\Omega$ with a Hilbert manifold structure, locally modeled on $L^{2}\left(I, \mathbb{R}^{2}\right)$. The endpoint map $F: \Omega \rightarrow M$ is the smooth map assigning to each curve its final point $F(\gamma)=\gamma(1)$. Given $y \in M$ we call $\Omega(y):=F^{-1}(y)$ the set of all horizontal curves joining $x_{0}$ and $y$. If $y$ is a regular value of $F$, then $\Omega(y)$ is a smooth Hilbert submanifold. In general, however, $y$ is not regular and $\Omega(y)$ is a submanifold with singularities.

${ }^{*}$ F.B. has been supported by the ANR SRGI (reference ANR-15-CE40-0018), and by the University of Padova STARS Project "Sub-Riemannian Geometry and Geometric Measure Theory Issues: Old and New".

${ }^{* *}$ F.B. and A.A.A. warmly thank the anonymous reviewer for the care paid in the revision process. Many parts of this text have been significantly polished and simplified thanks to his/her suggestions.

Keywords and phrases: Sub-Riemannian geometry, abnormals, endpoint mapping, normal forms.

1 SISSA (Trieste) and Steklov Institute, Moscow, Russia.

2 Dipartimento di Matematica Tullio Levi-Civita, Università degli studi di Padova, Padova, Italy.

*** Corresponding author: francesco.boarotto@gmail.com 
A curve $\gamma$ is singular (or abnormal) if $d_{\gamma} F: T_{\gamma} \Omega \rightarrow T_{F(\gamma)} M$ is not surjective. The corank of $\gamma$ is the codimension of $\operatorname{Im}\left(d_{\gamma} F\right)$ in $T_{F(\gamma)} M$. Singular curves are central objects in the theory of nonholonomic distributions, but their study is a difficult problem and many fundamental questions related to their structure are still open $[3,16]$. Most of the difficulties come from the fact that the Hessian of $F$ is always degenerate, and even in the simplest case of a singular curve $\gamma$ of corank one, it is difficult to obtain local information on $F$ from the Hessian: for example it is known [8] that the endpoint map is always locally open in the $W^{1,2}$-topology even if the Hessian has a sign.

Nevertheless, singular curves are interesting for the following reason. Fix on $\Delta$ a Riemannian metric, and denote by $\|\cdot\|_{L^{2}\left(I, \mathbb{R}^{2}\right)}$ the associated $L^{2}$ norm. Define then the energy functional $J: \Omega \rightarrow \mathbb{R}$ by the formula $J(\gamma)=\frac{1}{2}\|\dot{\gamma}\|_{L^{2}\left(I, \mathbb{R}^{2}\right)}^{2}$. The sub-Riemannian minimizing problem between $x_{0}$ and $y \in M$ consists in finding the admissible curves realizing $\min \{J(\gamma) \mid \gamma \in \Omega(y)\}$, and Montgomery proved for the first time in [15] that singular curves may be solutions to the problem (in fact, even independently on the choice of the metric).

\subsection{Regular singular curves}

Let $\mathcal{F}: \Omega \rightarrow M \times \mathbb{R}$ denote the extended endpoint map, that is $\mathcal{F}$ is the pair $(F, J)$. Candidate solutions $\gamma$ to the sub-Riemannian minimizing problem, called extremals, are constrained extremal points for $\mathcal{F}$ and in particular a singular curve is an extremal. An extremal is called strictly singular (or strictly abnormal) if, for every nonzero $\xi \in T_{\mathcal{F}(\gamma)}^{*}(M \times \mathbb{R})=T_{F(\gamma)}^{*} M \times \mathbb{R}$ such that $\xi d_{\gamma} \mathcal{F}=0$, the projection of $\xi$ onto its $\mathbb{R}$-factor is zero.

Let us define $\Delta^{2}:=[\Delta, \Delta]$ and $\Delta^{3}:=\left[\Delta, \Delta^{2}\right]$, where $[\cdot, \cdot]$ are the usual Lie brackets. Borrowing the terminology from [14], we say that a singular curve $\gamma$ is regular if the Pontryagin Maximum Principle provides a nowhere zero curve $\eta(t)$ (sometimes called biextremal in the literature), dual to $\gamma(t)$, such that $\eta(t) \in\left(\Delta_{\gamma(t)}^{2}\right)^{\perp} \backslash\left(\Delta_{\gamma(t)}^{3}\right)^{\perp}$ for every $t \in I$. Regular singular curves are the least degenerate singular curves for sub-Riemannian structures of rank 2, and it was proven in [14] that these curves are smooth (see also [7], Thm. 4.4). ${ }^{1}$

Let us fix a regular singular curve $\gamma \in \Omega(y)$. Our study of the endpoint map $F$ being local around $\gamma$, we assume without loss of generality that $\gamma$ does not have self-intersections, lifting if necessary both $\Delta$ and $\gamma$ to a covering space of a neighborhood $\mathcal{O}_{\operatorname{supp}(\gamma)} \subset M$ of $\operatorname{supp}(\gamma):=\{\gamma(t) \mid t \in I\}$. Then there are smooth vector fields $X_{1}$ and $X_{2}$ such that $\Delta_{x}=\operatorname{span}\left\{X_{1}(x), X_{2}(x)\right\}$ for every $x \in \mathcal{O}_{\operatorname{supp}(\gamma)}$, and such that $\gamma$ is an integral curve of the field $X_{1}$; in particular, admissible curves contained in $\mathcal{O}_{\operatorname{supp}(\gamma)}$ are parameterized by the solutions to the differential system:

$$
\dot{x}(t)=\left(1+v_{1}(t)\right) X_{1}(x(t))+v_{2}(t) X_{2}(x(t)), \quad x(0)=x_{0}, \quad \text { a.e. } t \in I
$$

with $v=\left(v_{1}, v_{2}\right) \in L^{2}\left(I, \mathbb{R}^{2}\right)$. Equation (1.2) identifies an admissible curve $x$ with its control $v$ and, with a slight abuse of notation, it allows to treat $F$ as a map on the space of controls, in such a way that $F(0)=F(\gamma)$.

\subsection{Rank-two nice singular curves and main results}

Assume that $\gamma$ is a corank-one regular singular curve in $\Omega(y)$, and let $t \mapsto \lambda(t)$ be the associated abnormal extremal, normalized so that $\lambda=\lambda(1) \in T_{y}^{*} M$ has norm one. Then the Hessian of $F$ at $\gamma$ is given by the quadratic form $\lambda \mathrm{He}_{0} F: \operatorname{ker}\left(d_{0} F\right) \rightarrow \mathbb{R}$.

Much in the spirit of the classical Morse theory, we would like to exploit the Hessian $\lambda \mathrm{He}_{0} F$ to find a normal form for $F$ around $\gamma$. There are two main issues that we need to discuss:

$-\lambda \mathrm{He}_{0} F$ is highly degenerate: in the coordinate system defined by (1.2), $\lambda \mathrm{He}_{0} F$ does not depend on the $v_{1}$-coordinate, and all the controls of the form $\left(v_{1}, 0\right)$ with $v_{1}$ of zero mean belong to its kernel;

- The eigenvalues of $\lambda \mathrm{He}_{0} F$ might accumulate towards zero in the $L^{2}\left(I, \mathbb{R}^{2}\right)$ topology.

\footnotetext{
${ }^{1}$ We recall that a generic (with respect to the $C^{\infty}$-Whitney topology on the set of distributions $\Delta$ ) sub-Riemannian structure of rank bigger than 2 has no regular singular curves by Corollary 2.5 of [9].
} 
For the first point we limit our considerations to the vector subspace $L^{\infty}(I, \mathbb{R}) \oplus L^{2}(I, \mathbb{R})$. To deal with the second, we restrict our study only to a special subclass of abnormal curves, that we define later in Definition 1.2, and we rely on the strong Legendre condition (see Prop. 4.4): namely, we exploit the fact that, up to change the sign of $\lambda$ there exists $\kappa>0$ such that

$$
\left\langle\lambda(t),\left[X_{2},\left[X_{1}, X_{2}\right]\right](\gamma(t))\right\rangle>\kappa \text { for every } t \in I .
$$

It is not immediate, however, to see how the Legendre condition appears into $\lambda \mathrm{He}_{0} F$. In fact, to use (1.3) we need to perform a Goh transformation on the $v_{2}$-component of the control and extend locally $F$ to a new map $\stackrel{\circ}{F}$ on $L^{\infty}(I, \mathbb{R}) \oplus \mathbb{R} \oplus L^{2}(I, \mathbb{R})$, which will be precisely introduced later in Definition 5.2. It is for the extended map $\stackrel{\circ}{F}$ that we can find a normal form, provided $y$ is not conjugate along $\gamma$, i.e. if the kernel of $\lambda \mathrm{He}_{0} \stackrel{\circ}{F}$ contains only the controls $\left(v_{1}, 0,0\right)$ with $v_{1}$ of zero mean.

Remark 1.1. Condition (1.3) is fundamental. Indeed, once we factor out $\operatorname{ker}\left(\lambda \mathrm{He}_{0} \stackrel{\circ}{F}\right)$, it allows for a decomposition of the Hessian as the sum of a coercive operator plus a compact perturbation.

This is not true for $\lambda \mathrm{He}_{0} F$, for which we cannot guarantee that its eigenvalues do not accumulate towards zero, not even after the factorization of the kernel.

Definition 1.2. An admissible singular curve $\gamma$ is a rank-two-nice singular curve if

(i) $\gamma$ is a corank-one regular strictly singular curve.

(ii) $y=F(\gamma)$ is not a conjugate point along $\gamma$. The precise meaning of this condition is rather technical, and it has only been hinted at right before Remark 1.1. It will be presented with all the due details later on in Definition 4.2, and discussed further in the subsequent Remark.

Notice that $L^{\infty}(I, \mathbb{R}) \oplus \mathbb{R} \oplus L^{2}(I, \mathbb{R})$ admits the orthogonal direct sum decomposition

$$
L^{\infty}(I, \mathbb{R}) \oplus \mathbb{R} \oplus L^{2}(I, \mathbb{R})=\operatorname{ker}\left(d_{0} \stackrel{\circ}{F}\right) \oplus \stackrel{\circ}{E},
$$

where $\stackrel{\circ}{E}$ is a vector subspace of finite codimension isomorphic to $\operatorname{Im}\left(d_{0} \stackrel{\circ}{F}\right)$ via $d_{0} \stackrel{\circ}{F}$. Accordingly, we will write coordinates $z=\left(z^{\prime}, z^{\prime \prime}\right)$ adapted to this splitting.

Theorem 1.3. Let $\gamma \in \Omega(y)$ be a rank-two-nice singular curve. Then there exist a neighborhood of the origin $\mathcal{W} \subset \operatorname{ker}\left(d_{0} \stackrel{\circ}{F}\right) \oplus \stackrel{\circ}{E}$, an origin-preserving diffeomorphism $\mu: \mathcal{W} \rightarrow \mathcal{W}$, and a diffeomorphism $\psi: \mathcal{O}_{y} \rightarrow \mathcal{O}_{0}$ of neighborhoods $\mathcal{O}_{y} \subset M$ and $\mathcal{O}_{0} \subset \mathbb{R} \oplus \operatorname{Im}\left(d_{0} \stackrel{\circ}{F}\right)$, respectively of $y$ and 0 , such that

$$
\psi \circ \stackrel{\circ}{F} \circ \mu\left(z^{\prime}, z^{\prime \prime}\right)=\left(\lambda \mathrm{He}_{0} \stackrel{\circ}{F}\left(z^{\prime}\right), d_{0} \stackrel{\circ}{F}\left(z^{\prime \prime}\right)\right)
$$

for every $\left(z^{\prime}, z^{\prime \prime}\right) \in \mathcal{W}$.

We notice at this point that the restriction to the space $L^{\infty}(I, \mathbb{R}) \oplus L^{2}(I, \mathbb{R})$ is not by chance, and indeed a normal form could not exist on the whole $L^{2}\left(I, \mathbb{R}^{2}\right)$. On the one hand, indeed, for every rank-two-nice curve $\gamma$ the negative eigenspace $N$ of the Hessian $\lambda \mathrm{He}_{0} F$ is finite dimensional, and possibly empty (see Proposition 4.7). On the other hand it is known ([8], Prop. 2) that the restriction of $F$ to any subspace of finite codimension is an open map. Were a normal form as in Theorem 1.3 possible on $L^{2}\left(I, \mathbb{R}^{2}\right)$, on the orthogonal complement of $N$ the projection of $F$ along the "abnormal" direction would have the sign of $\lambda \mathrm{He}_{0} F$, providing a contradiction.

The fact of having a normal form allows for a finer analysis of $\Omega(y)$ locally around $\gamma$, as demonstrated by the following result.

Proposition 1.4. Let $\gamma \in \Omega(y)$ be a rank-two-nice singular curve. There exists a weak neighborhood of the origin $\mathcal{U} \subset L^{2}\left(I, \mathbb{R}^{2}\right)$, such that the only singular controls contained in $\mathcal{U}$ are of the form $v=\left(v_{1}, 0\right)$, with $v_{1}$ of zero mean.

As a consequence, $\gamma$ is isolated among singular curves contained in $\Omega(y)$ with respect to the $W^{1,2}$-topology. 

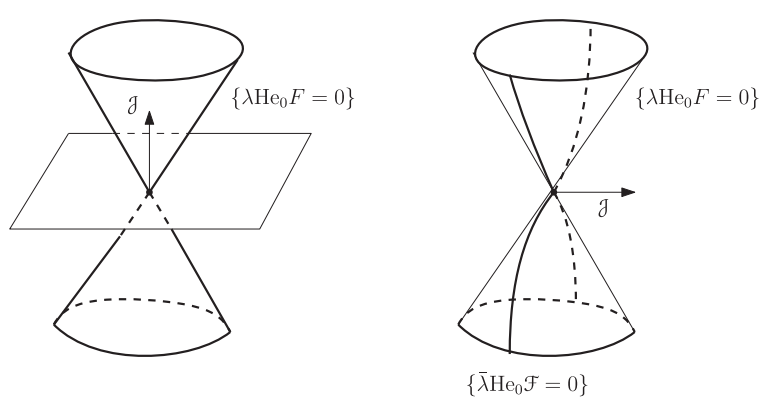

Figure 1 . The relative positions between $\mathcal{J}:=\pi_{\operatorname{ker}\left(d_{0} F\right)} \nabla_{0} J$ and the quadratic cone $\left\{\lambda \mathrm{He}_{0} F=\right.$ $0\}$. In the first case $\lambda \mathrm{He}_{0} F$ and $\bar{\lambda} \mathrm{He}_{0} \mathcal{F}$ have different indexes, in the second the indexes are the same.

It would be nice to know if Theorem 1.3 allows to conclude for the isolation of $\gamma$ even among normal extremals. At the moment, this is not clear to the authors, for this investigation would require more work in proving that Theorem 1.3 induces a normal form even on $L^{\infty}(I, \mathbb{R}) \oplus L^{2}(I, \mathbb{R})$. Nevertheless Theorem 1.3 makes evident that the topological information provided by a rank-two-nice curve $\gamma$ is contained

- in the pair $\left(\operatorname{ind}\left(\lambda \mathrm{He}_{0} F\right)\right.$, ind $\left.\left(\bar{\lambda} \mathrm{He}_{0} \mathcal{F}\right)\right)$, where $\bar{\lambda}=(\lambda, 0)$. By construction, we have

$$
\operatorname{ind}\left(\bar{\lambda} \mathrm{He}_{0} \mathcal{F}\right) \leq \operatorname{ind}\left(\lambda \mathrm{He}_{0} F\right) \leq \operatorname{ind}\left(\bar{\lambda} \mathrm{He}_{0} \mathcal{F}\right)+1 ;
$$

- in the relative position of $\mathcal{J}:=\pi_{\operatorname{ker}\left(d_{0} F\right)} \nabla_{0} J$ with respect to the cone $\left\{\lambda \mathrm{He}_{0} F=0\right\}$, as shown in Figure 1.

We expect that rank-two-nice curves have an influence on the homotopy type of the Lebesgue sets of $J$, i.e. of the sets $\{J \leq c\}$ for $c \in \mathbb{R}$, and we plan to study how the "homotopical (in)visibility" (in the $W^{1,2}$-topology) of a rank-two-nice curve $\gamma$ is affected by the interplay between $\operatorname{ind}\left(\lambda \mathrm{He}_{0} F\right)$ and $\operatorname{ind}\left(\bar{\lambda} \mathrm{He}_{0} \mathcal{F}\right)$ in a forthcoming paper, completing the Morse-like results contained in [4] to the case of sub-Riemannian structures of rank two.

We only recall that, if there are no conjugate points along $\gamma$ between $x_{0}$ and $y$, it is known by [7] that $\gamma$ is indeed a local minimizer in the $W^{1,2}$-topology. This result, however, is proved by showing that the perturbation provided by $\lambda \mathrm{He}_{0} F$ along the abnormal direction is not compensated by higher order terms, and does not require any normal form for $F$.

\subsection{An explicit computation of conjugate times}

Let $M=S O(3) \times \mathbb{R}$ and $\mathfrak{m}=\mathfrak{s o}(3) \oplus \mathbb{R}$ be its Lie algebra. Let

$$
X_{1}:=\frac{\left(T_{1}+T_{2}\right) \oplus 2}{\sqrt{2}}, \quad X_{2}:=\frac{T_{1} \oplus 1}{\sqrt{2}}
$$

where $T_{1}, T_{2}, T_{3}$ are the standard generators for $\mathfrak{s o}(3)$, that is $\left[T_{1}, T_{2}\right]=T_{3},\left[T_{2}, T_{3}\right]=T_{1},\left[T_{3}, T_{1}\right]=T_{2}$.

We define a distribution $\Delta \subset T M$ extending these vectors to smooth vector fields on $M$ by left-translation and we consider on $\Delta$ the metric that makes $X_{1}$ and $X_{2}$ orthonormal. The energy of a horizontal curve is the squared $L^{2}$-norm of its velocity.

We consider $\gamma$ to be an integral curve of $X_{1}$. This curve satisfies point (i) in Definition 1.2 by the results in Section 8 of [21]. We denote by $\gamma_{s}:=\left.\gamma\right|_{[0, s]}$ the restriction of $\gamma$ to the interval $[0, s]$. 


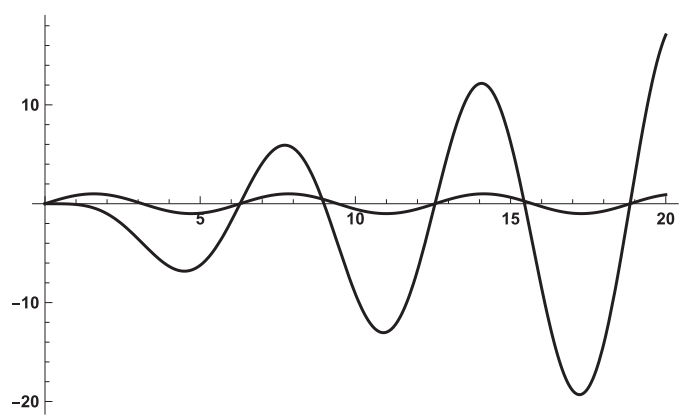

Figure 2. Plot of the functions $a_{F}(s)$ and $a_{\mathcal{F}}(s)$.

The set of times $s \in I$ for which $\gamma(s)$ is a conjugate point along $\gamma$ for the map $F$ (resp. for the map $\mathcal{F}$, i.e., for the map $F$ restricted onto the level sets of $J$ ) is given by the sets $\left\{a_{F}=0\right\}$ (resp. $\left\{a_{\mathcal{F}}=0\right\}$ ), where:

$$
\begin{aligned}
& a_{F}(s)=\sin (s), \\
& a_{\mathcal{F}}(s)=s \sin (s)+2(\cos (s)-1) .
\end{aligned}
$$

We will explain later at the end of Section 6.4 how to derive such equations.

The mapping $s \mapsto\left(\operatorname{ind}\left(\lambda(s) \mathrm{He}_{\gamma_{s}} F\right)\right.$, ind $\left.\left(\bar{\lambda}(s) \mathrm{He}_{\gamma_{s}} \mathcal{F}\right)\right)$ is piecewise constant on $I$ : the value of $\operatorname{ind}\left(\lambda(s) \mathrm{He}_{\gamma_{s}} F\right)$ (resp. of ind $\left(\bar{\lambda}(s) \mathrm{He}_{\gamma_{s}} \mathcal{F}\right)$ ) changes when $s$ is a zero of $a_{F}$ (resp. a zero of $a_{\mathcal{F}}$ ). In particular, there exist time intervals arbitrarily far from zero on which either $\lambda(s) \mathrm{He}_{\gamma_{s}} F$ and $\bar{\lambda}(s) \mathrm{He}_{\gamma_{s}} \mathcal{F}$ have the same index, or where these indexes differ by one. Looking at Figure 2, we see that the initial values of $\left(\operatorname{ind}\left(\lambda(s) \mathrm{He}_{\gamma_{s}} F\right)\right.$, ind $\left(\bar{\lambda}(s) \mathrm{He}_{\gamma_{s}} \mathcal{F}\right)$ ) are given by:

$$
(0,0),(1,0),(2,1),(2,2),(3,2),(4,3),(4,4),(5,4),(6,5), \ldots
$$

\subsection{Structure of the paper}

We give in Section 2 all the technical preliminaries, and we study the endpoint map $F$ at the first and at the second order. We introduce the space $L^{\infty}(I, \mathbb{R}) \oplus L^{2}(I, \mathbb{R})$ in Section 3 , where we provide the reparametrization map which is needed to factor out the kernel of the Hessian. The extension to the map $\stackrel{\circ}{F}$, along with the notion of conjugate points, is discussed in Section 4. We also establish in this section some useful analytical properties of $\lambda \mathrm{He}_{0} \stackrel{\circ}{F}$. Section 5 is the core of the paper, where we prove the existence of a local normal form for the endpoint map $\stackrel{\circ}{F}$ and prove Theorem 1.3 and Proposition 1.4. Finally, in Section 6 we characterize conjugate points along $\gamma$ on the cotangent space $T^{*} M$ in terms of an appropriate Jacobi equation. We study in details the class of Engel structures, completing the explanation of our previous example.

\section{RANK-TWO SUB-RIEMANNIAN STRUCTURES}

\subsection{Elements of chronological calculus}

We will use extensively in the paper the notations and the tools of chronological calculus, introduced in [1]. For the interested reader we refer to [5]; we report here only what we need to keep the article self-contained.

We identify points $x \in M$ as linear functionals on the algebra $C^{\infty}(M)$, that is as evaluations $x(a)=a(x)$, and we interpret smooth diffeomorphisms $P$ of $M$ as automorphisms of $C^{\infty}(M)$ defined by the formula $P(a(x))=$ $a(P(x))$. Finally, we identify a smooth vector field $X \in \operatorname{Vec}(M)$ with the derivation of the algebra $C^{\infty}(M)$ given by $a \mapsto X a$. 
A time-dependent vector field is a family $\left(X_{t}\right)_{t \in I}$ contained in $\operatorname{Vec}(M)$. We assume throughout the paper that the mapping $t \mapsto X_{t}$ for $t \in I$ is locally integrable in time. Then the flow $P_{t}$ of a time-dependent vector field $X_{t}$ is defined through the following Cauchy problem²:

$$
\left\{\begin{array}{l}
\dot{x}(t)=X_{t}(x(t)), \\
x(0)=x_{0}
\end{array}\right.
$$

In particular, the flow of a time-dependent vector field on $M$ is an absolutely continuous curve in the group of diffeomorphisms of $M, t \mapsto P_{t}$ for $t \in I$, with the property that $P_{0}$ is the identity map on $M$.

The Cauchy Problem (2.1) can be reformulated as the following Cauchy problem of operators on $C^{\infty}(M)$ :

$$
\dot{P}_{t}=P_{t} \odot X_{t}, \quad P_{0}=\mathrm{Id},
$$

where $\odot$ is the composition of operators on $C^{\infty}(M)$ acting from right to left, i.e.:

$$
\left(x_{0} \odot \dot{P}_{t}\right) a=\left(x_{0} \odot P_{t} \odot X_{t}\right) a=X_{t} a\left(P_{t}\left(x_{0}\right)\right)
$$

for every $a \in C^{\infty}(M)$ and every $x_{0} \in M$. The characterization (2.2) of $P_{t}$ motivates the following notation:

$$
\overrightarrow{\exp } \int_{0}^{t} X_{\tau} \mathrm{d} \tau:=P_{t}
$$

and we call $P_{t}$ the right chronological exponential of $X_{t}$. Equation (2.4) admits an infinite expansion as a Volterra series. We will only need the terms of order up to two in this expansion, that is ${ }^{3}$

$$
\overrightarrow{\exp } \int_{0}^{t} X_{\tau} \mathrm{d} \tau=\mathrm{Id}+\int_{0}^{t} X_{\tau_{1}} \mathrm{~d} \tau_{1}+\iint_{0 \leq \tau_{2} \leq \tau_{1} \leq t} X_{\tau_{2}} \odot X_{\tau_{1}} \mathrm{~d} \tau_{2} \mathrm{~d} \tau_{1}+\ldots
$$

We denote by $Q_{t}$ the inverse of $P_{t}$. Differentiating the relation $P_{t} \odot Q_{t}=\operatorname{Id}$ we find $\dot{Q}_{t}=-X_{t} \odot Q_{t}$. Therefore we set

$$
\overleftarrow{\exp } \int_{0}^{t}\left(-X_{\tau}\right) \mathrm{d} \tau:=Q_{t}
$$

and we call $Q_{t}$ the left chronological exponential of $-X_{t}$.

For a diffeomorphism $P$ of $M$ we adopt the notation $\mathrm{Ad} P$ to denote the following inner automorphism of the Lie algebra $\operatorname{Vec}(M)$ :

$$
\operatorname{Ad} P: X \mapsto P \odot X \odot P^{-1}=P_{*}^{-1} X
$$

where this last notation stands for the result of the translation of $X$ via the differential of $P^{-1}$. These notions apply in particular to the maps $\operatorname{Ad} P_{t}$, allowing for the following "infinitesimal" characterization: for every $Y \in \operatorname{Vec}(M)$ there holds

$$
\frac{\mathrm{d}}{\mathrm{d} t}\left(\operatorname{Ad} P_{t}\right) Y=\left(\operatorname{Ad} P_{t}\right)\left[X_{t}, Y\right]=:\left(\operatorname{Ad} P_{t}\right) \operatorname{ad}\left(X_{t}\right) Y,
$$

\footnotetext{
${ }^{2}$ We assume for our purposes that the solutions to the Cauchy problem are always defined for every $t \in I$.

${ }^{3}$ If $X$ is time-independent this expansion coincides with the well-known formula $e^{t X}=\operatorname{Id}+t X+\frac{t^{2}}{2} X^{2}+\ldots$
} 
where, by definition, $\operatorname{ad}(X) Y:=[X, Y]$ denotes the (left) Lie-bracket as an operator on $\operatorname{Vec}(M)$. We then see that $\operatorname{Ad} P_{t}$ is the unique solution to the Cauchy problem for operators on $\operatorname{Vec}(M)$

$$
\dot{A}_{t}=A_{t} \odot \operatorname{ad} X_{t}, \quad A_{0}=\mathrm{Id} .
$$

This motivates the following notation:

$$
\overrightarrow{\exp } \int_{0}^{t} \operatorname{ad} X_{\tau} \mathrm{d} \tau:=\operatorname{Ad}\left(\overrightarrow{\exp } \int_{0}^{t} X_{\tau} \mathrm{d} \tau\right)
$$

Equation (2.7) admits as well an expansion as a formal series that, up to the second order, reads

$$
\overrightarrow{\exp } \int_{0}^{t} \operatorname{ad} X_{\tau} \mathrm{d} \tau=\operatorname{Id}+\int_{0}^{t} \operatorname{ad} X_{\tau_{1}} \mathrm{~d} \tau_{1}+\iint_{0 \leq \tau_{2} \leq \tau_{1} \leq t} \operatorname{ad} X_{\tau_{2}} \odot \operatorname{ad} X_{\tau_{1}} \mathrm{~d} \tau_{2} \mathrm{~d} \tau_{1}+\ldots
$$

Chronological calculus provides a non-autonomous version of the classical "variation of the constants' formula":

$$
\begin{aligned}
\overrightarrow{\exp } \int_{0}^{t}\left(X_{\tau}+Y_{\tau}\right) \mathrm{d} \tau & =\overrightarrow{\exp } \int_{0}^{t} X_{\tau} \mathrm{d} \tau \odot \overrightarrow{\exp } \int_{0}^{t}\left(\overrightarrow{\exp } \int_{t}^{\tau} \operatorname{ad} X_{\theta} \mathrm{d} \theta\right) Y_{\tau} \mathrm{d} \tau \\
& =\overrightarrow{\exp } \int_{0}^{t}\left(\overrightarrow{\exp } \int_{0}^{\tau} \operatorname{ad} X_{\theta} \mathrm{d} \theta\right) Y_{\tau} \mathrm{d} \tau \odot \overrightarrow{\exp } \int_{0}^{t} X_{\tau} \mathrm{d} \tau
\end{aligned}
$$

which also allows to differentiate with respect to the parameter $\varepsilon$ a non-autonomous vector field. Specifically, given $X_{t}(\varepsilon) \in \operatorname{Vec}(M)$ we have

$$
\frac{\partial}{\partial \varepsilon} \overrightarrow{\exp } \int_{0}^{1} X_{t}(\varepsilon) \mathrm{d} t=\overrightarrow{\exp } \int_{0}^{1} X_{t}(\varepsilon) \mathrm{d} t \odot \int_{0}^{1}\left(\overrightarrow{\exp } \int_{1}^{t} \operatorname{ad} X_{\tau}(\varepsilon) \mathrm{d} \tau\right) \frac{\partial}{\partial \varepsilon} X_{t}(\varepsilon) \mathrm{d} t
$$

For a rigorous derivation of (2.10) we refer to Section 2.8 of [5].

\subsection{Technical preliminaries}

Let $\Omega$ be defined as in (1.1). For $t \in I$, the map $F_{t}$ is the map that returns the point $\gamma(t)$ of a horizontal curve $\gamma \in \Omega$ :

$$
F_{t}: \Omega \rightarrow M, \quad F_{t}(\gamma)=\gamma(t)
$$

Then $F=F_{1}$ is the endpoint map. We recall in the following proposition some useful properties of $F_{t}$ (see $[8,22])$.

Proposition 2.1. For every $t \in I$, the map $F_{t}: \Omega \rightarrow M$ is smooth with respect to the Hilbert manifold structure on $\Omega$. Moreover, if $\gamma_{n} \rightarrow \gamma$ weakly, then $F_{t}\left(\gamma_{n}\right) \rightarrow F_{t}(\gamma)$ and $d_{\gamma_{n}} F_{t} \rightarrow d_{\gamma} F_{t}$ in the operator norm, uniformly with respect to $t$.

Definition 2.2. We say that $\gamma \in \Omega$ is a singular (or abnormal) curve if $\gamma$ is a critical point of $F$, or equivalently if $d_{\gamma} F: T_{\gamma} \Omega \rightarrow T_{F(\gamma)} M$ is not surjective. The corank of $\gamma$ is the codimension of the image $\operatorname{Im}\left(d_{\gamma} F\right)$ of $d_{\gamma} F$ in $T_{F(\gamma)} M$.

We fix a Riemannian metric $g$ on $\Delta$, and we introduce the energy functional $J: \Omega \rightarrow \mathbb{R}$ by the formula

$$
J(\gamma)=\frac{1}{2} \int_{0}^{1} g(\dot{\gamma}(t), \dot{\gamma}(t)) \mathrm{d} t
$$


Definition 2.3. We define the extended endpoint map $\mathcal{F}: \Omega \rightarrow M \times \mathbb{R}$ by

$$
\mathcal{F}(\gamma)=(F(\gamma), J(\gamma))
$$

Given a point $y \in M$ different from $x_{0}$, we consider the problem of finding the admissible curves $\gamma$ connecting $x_{0}$ and $y$ that minimize the energy $J$. This problem can be reformulated as a constrained minimum problem on $\mathcal{F}$ : a curve $\gamma$ is a candidate minimizer if there exists a nonzero covector $\xi=\left(\lambda, \lambda_{0}\right) \in T_{F(\gamma)}^{*} M \times \mathbb{R}$, defined up to scalar multiples, such that

$$
\xi d_{\gamma} \mathcal{F}=\lambda d_{\gamma} F+\lambda_{0} d_{\gamma} J=0
$$

Candidate minimizing curves are called extremals, and the Pontryagin Maximum Principle [18] (PMP, in short) characterizes extremals in terms of nowhere zero, absolutely continuous curves $\eta: I \rightarrow T^{*} M \times \mathbb{R}$, called biextremals or extremal lifts: an admissible curve $\gamma$ is an extremal only if it is the projection on $M$ of a biextremal. ${ }^{4}$

Notice that the initial datum $\lambda \in T_{F(\gamma)}^{*} M$ of every biextremal curve $\eta$ is the first component of a Lagrange multiplier $\xi=\left(\lambda, \lambda_{0}\right)$ in (2.14). If $\xi$ is such that $\lambda_{0}=-1$, we say that $\eta$ is a normal biextremal and $\gamma$ is a normal extremal curve. In this case small pieces of $\gamma$ are geodesics in the classical sense, i.e. sufficiently short pieces of $\gamma$ are minimizing curves between their endpoints. If instead $\lambda_{0}=0, \gamma$ is a singular curve and it is the projection of an abnormal biextremal starting at $\lambda$, as we will now explain.

The cotangent bundle $T^{*} M$ is canonically endowed with a symplectic form $\omega$, that is a closed non-degenerate smooth section of $\Lambda^{2}\left(T^{*} M\right)$, and a bundle projection $\pi: T^{*} M \rightarrow M$. Consider the subspace

$$
\Delta^{\perp}:=\left\{\lambda \in T^{*} M \mid\langle\lambda, v\rangle=0 \text {, for every } v \in \Delta\right\} \subset T^{*} M,
$$

where the notation $\langle\cdot, \cdot\rangle$ stands for the duality product between vectors and covectors. The restriction $\bar{\omega}$ of $\omega$ to $\Delta^{\perp}$ no longer needs to be non-degenerate and may admit characteristic lines [16].

Definition 2.4. A nowhere zero, absolutely continuous curve $\eta: I \rightarrow \Delta^{\perp}$ is an abnormal biextremal if, for a.e. $t \in I, \dot{\eta}(t)$ belongs to $\operatorname{ker}\left(\bar{\omega}_{\eta(t)}\right)$, that is if for a.e. $t \in I$,

$$
\bar{\omega}_{\eta(t)}(\dot{\eta}(t), \nu)=0
$$

for every $\nu \in T_{\eta(t)} \Delta^{\perp}$.

Proposition 2.5 ([13], Thm. 10). An admissible curve $\gamma \in \Omega$ is singular if and only if $\gamma$ is the projection of an abnormal biextremal $\eta: I \rightarrow \Delta^{\perp}$. As a matter of terminology, we say that $\eta$ is an abnormal lift of $\gamma$.

An admissible curve $\gamma$ may be at the same time both normal and singular, and we say that an admissible curve $\gamma$ is strictly singular (or strictly abnormal) if, for every $\xi=\left(\lambda, \lambda_{0}\right) \in T_{F(\gamma)}^{*} M \times \mathbb{R}$ such that (2.14) holds, $\lambda_{0}=0$. For a further discussion on these points, we refer e.g. to $[5,18,19]$.

Let now $\Delta$ be a distribution of rank 2 , and $\gamma \in \Omega$ be a singular curve. Then it is well-known ([2], Sect. 12.4) that for every abnormal lift $\eta: I \rightarrow \Delta^{\perp}$ of $\gamma$ the Goh condition,

$$
\langle\eta(t),[X, Y](\gamma(t))\rangle=0,
$$

holds for every $t \in I$ and every pair $X, Y$ of local smooth sections of $\Delta$, that is $\eta(t) \in\left(\Delta_{\gamma(t)}^{2}\right)^{\perp}$ for every $t \in I$.

\footnotetext{
${ }^{4}$ The classical PMP is stated in terms of $W^{1, \infty}$-curves. There are, however, no complications for $W^{1,2}$-curves since every admissible curve might be parameterized by the length of arc ([2], Sect. 3.6).
} 
Definition 2.6. We say that $\gamma \in \Omega$ is a regular singular curve if $\gamma$ has an abnormal lift $\eta: I \rightarrow \Delta^{\perp}$ satisfying

$$
\eta(t) \in\left(\Delta_{\gamma(t)}^{2}\right)^{\perp} \backslash\left(\Delta_{\gamma(t)}^{3}\right)^{\perp}
$$

for every $t \in I$.

Regular singular curves are smooth, see e.g. [14] or Theorem 4.4 of [7]. Moreover, for every regular singular curve $\gamma$, there exists $0<s \leq 1$ such that $\gamma_{s}:=\left.\gamma\right|_{[0, s]}$ is a strict local minimizer for the $W^{1,2}$-topology on the space of admissible curves joining $x_{0}$ and $\gamma(s)$. This property depends just on the sub-Riemannian structure $(M, \Delta)$, and not on the metric chosen on it.

\subsubsection{Adapted coordinates}

We briefly explain how to put coordinates on $\Omega$, locally around a regular singular curve $\gamma$. We assume as in (1.2) that there exist a neighborhood $\mathcal{O}_{\operatorname{supp}(\gamma)} \subset M$ of $\gamma$ and $X_{1}, X_{2} \in \operatorname{Vec}(M)$ such that:

- $\gamma$ is an integral curve of $X_{1}$ associated with the control $(1,0)$, satisfying $\dot{\gamma}(t)=X_{1}(\gamma(t))$ for every $t \in I$.

$-\Delta_{x}=\operatorname{span}\left\{X_{1}(x), X_{2}(x)\right\}$, for every $x \in \mathcal{O}_{\operatorname{supp}(\gamma)}$.

Horizontal curves $\gamma^{\prime}$ contained in $\mathcal{O}_{\operatorname{supp}(\gamma)}$ are described a.e. on $I$ by the solutions of the differential system

$$
\dot{\gamma}^{\prime}(t)=u_{1}(t) X_{1}\left(\gamma^{\prime}(t)\right)+u_{2}(t) X_{2}\left(\gamma^{\prime}(t)\right), \quad \gamma^{\prime}(0)=x_{0},
$$

where $u \in \mathcal{U}_{1} \subset L^{2}\left(I, \mathbb{R}^{2}\right)$ and the open set $\mathcal{U}_{1} \subset L^{2}\left(I, \mathbb{R}^{2}\right)$ is a neighborhood of $(1,0)$ that consists of all the pairs $\left(u_{1}, u_{2}\right)$ such that the solution to (2.19) exists for every $t \in I$.

Additionally, for every $x \in \mathcal{O}_{\operatorname{supp}(\gamma)}$ we endow $\Delta_{x}$ with the Riemannian metric $g_{x}$ that makes $X_{1}(x)$ and $X_{2}(x)$ orthonormal. Then we see from (2.12) that

$$
J\left(\gamma^{\prime}\right)=\frac{1}{2} \int_{0}^{1}\left|u_{1}(t)\right|^{2}+\left|u_{2}(t)\right|^{2} \mathrm{~d} t
$$

Definition 2.7. A local chart on $\mathcal{U}_{1}$ is the choice of a neighborhood $\mathcal{V}_{1} \subset L^{2}\left(I, \mathbb{R}^{2}\right)$ of zero and a system of coordinates

$$
\left(u_{1}, u_{2}\right) \mapsto\left(1+v_{1}, v_{2}\right)
$$

on $\mathcal{U}_{1}$ and centered at $(1,0)$.

With the choice of a local chart, admissible curves are in one-to-one correspondence with integral curves of

$$
\dot{x}(t)=\left(1+v_{1}(t)\right) X_{1}(x(t))+v_{2}(t) X_{2}(x(t)), \quad x(0)=x_{0},
$$

for a.e. $t \in I$ and $v=\left(v_{1}, v_{2}\right) \in \mathcal{V}_{1}$.

Let $A: \mathcal{V}_{1} \rightarrow \Omega$ be the map that associates to the pair $\left(v_{1}, v_{2}\right) \in \mathcal{V}_{1}$ the only solution, up to zero-measure sets, to (2.22). Then $A: \mathcal{V}_{1} \rightarrow A\left(\mathcal{V}_{1}\right)$ is a smooth diffeomorphism, i.e., a local chart of $\Omega$ and, slightly abusing of the notation, we define on $\mathcal{V}_{1}$

$$
F(v):=F(A(v)) \text { and } J(v):=J(A(v)),
$$


where $F(v)$ and $J(v)$ are given by:

$$
\begin{aligned}
& F\left(v_{1}, v_{2}\right)=x_{0} \odot \overrightarrow{\exp } \int_{0}^{1}\left(1+v_{1}(t)\right) X_{1}+v_{2}(t) X_{2} \mathrm{~d} t, \quad \text { and } \\
& J\left(v_{1}, v_{2}\right)=\frac{1}{2} \int_{0}^{1}\left(1+v_{1}(t)\right)^{2}+v_{2}(t)^{2} \mathrm{~d} t .
\end{aligned}
$$

Definition 2.8. We say that a control $v \in \mathcal{V}_{1}$ is singular if

$$
d_{v} F=d_{A(v)} F \circ d_{v} A
$$

is not surjective. The corank of $v$ is the codimension of $\operatorname{Im}\left(d_{v} F\right)$ in $T_{F(v)} M$.

\subsection{The endpoint map near regular strictly singular curves}

Let $\gamma \in \Omega(y)$ be a reference regular strictly singular curve, and let us choose local coordinates centered at $(1,0)$, so that $\gamma$ becomes an integral curve of $X_{1}$ starting at $x_{0}$.

Locally around $\gamma$, by $(2.9)$, the endpoint map $F\left(v_{1}, v_{2}\right)$ can be seen as a perturbation of $y=F(0)$. Setting

$$
g_{t}:=e_{*}^{(1-t) X_{1}} X_{2}, \quad t \in I,
$$

we write:

$$
\begin{aligned}
F\left(v_{1}, v_{2}\right) & =x_{0} \odot \overrightarrow{\exp } \int_{0}^{1}\left(1+v_{1}(t)\right) X_{1}+v_{2}(t) X_{2} \mathrm{~d} t \\
& =y \odot \overrightarrow{\exp } \int_{0}^{1} v_{1}(t) X_{1}+v_{2}(t) g_{t} \mathrm{~d} t .
\end{aligned}
$$

\subsubsection{First-order conditions}

We see from (2.26) that the differential $d_{0} F: T_{0} \mathcal{V}_{1} \simeq L^{2}\left(I, \mathbb{R}^{2}\right) \rightarrow T_{F(0)} M$ is given by (see [5], Sect. 4):

$$
d_{0} F(v)=y \odot\left(\int_{0}^{1} v_{1}(t) d t X_{1}+\int_{0}^{1} v_{2}(t) g_{t} \mathrm{~d} t\right)=y \odot\left(\bar{v}_{1} X_{1}+\int_{0}^{1} v_{2}(t) g_{t} \mathrm{~d} t\right)
$$

for every $v \in L^{2}\left(I, \mathbb{R}^{2}\right)$. Here $\bar{v}_{1}$ denotes the integral mean of $v_{1}$ on $I$.

We orthogonally split the space of controls $L^{2}\left(I, \mathbb{R}^{2}\right)$ as the sum $\operatorname{ker}\left(d_{0} F\right) \oplus E$, where $E$ is a finite-dimensional complement of $\operatorname{ker}\left(d_{0} F\right)$, isomorphic to $\operatorname{Im}\left(d_{0} F\right)$ via the differential $d_{0} F$.

Lemma 2.9. Let $\gamma$ be a regular strictly singular curve. Then there exists $v_{2}^{0} \in L^{2}(I, \mathbb{R})$ such that $\operatorname{ker}\left(d_{0} F\right)$ admits the following $L^{2}\left(I, \mathbb{R}^{2}\right)$-orthogonal decomposition:

$$
\operatorname{ker}\left(d_{0} F\right)=Z_{1} \oplus \mathbb{R} \cdot\left(1,-v_{2}^{0}\right) \oplus Z_{2} .
$$

In particular every element $\left(v_{1}, v_{2}\right) \in \operatorname{ker}\left(d_{0} F\right)$ can be uniquely decomposed as

$$
\left(v_{1}, v_{2}\right)=\underbrace{\left(v_{1}-\bar{v}_{1}, 0\right)}_{Z_{1}}+\underbrace{\bar{v}_{1}\left(1,-v_{2}^{0}\right)}_{\mathbb{R} \cdot\left(1,-v_{2}^{0}\right)}+\underbrace{\left(0, v_{2}+\bar{v}_{1} v_{2}^{0}\right)}_{Z_{2}},
$$


and all the controls contained in $Z_{1}$ have zero mean. Similarly, for the extended endpoint map $\mathcal{F}$ we have the $L^{2}\left(I, \mathbb{R}^{2}\right)$-orthogonal decomposition:

$$
\operatorname{ker}\left(d_{0} \mathcal{F}\right)=\operatorname{ker}\left(d_{0} F\right) \cap \operatorname{ker}\left(d_{0} J\right)=Z_{1} \oplus Z_{2} .
$$

Proof. Recall that there exists $\lambda \in T_{y}^{*} M$ such that $\lambda d_{0} F=d_{0} J$ if and only if $\operatorname{ker}\left(d_{0} F\right) \subset \operatorname{ker}\left(d_{0} J\right)$. Since $\gamma$ is strictly singular by assumption, then $\left.d_{0} J\right|_{\operatorname{ker}\left(d_{0} F\right)} \neq 0$. If we combine this fact with the equalities

$$
\begin{aligned}
d_{0} J\left(v_{1}, v_{2}\right) & =\bar{v}_{1}, \\
d_{0} F\left(v_{1}, v_{2}\right) & =y \odot\left(\bar{v}_{1} X_{1}+\int_{0}^{1} v_{2}(t) g_{t} \mathrm{~d} t\right),
\end{aligned}
$$

we conclude that there must be in $\operatorname{ker}\left(d_{0} F\right)$ an element of the form $\left(1,-v_{2}^{0}\right)$, i.e., that there exists $v_{2}^{0} \in L^{2}(I, \mathbb{R})$ such that $d_{0} F\left(0, v_{2}^{0}\right)=X_{1}(y)$. Since $\left(1,-v_{2}^{0}\right) \notin \operatorname{ker}\left(d_{0} J\right),(2.28)$ and (2.29) readily follow.

\subsubsection{Second-order conditions}

We introduce in this section the Hessian map

$$
\begin{aligned}
\operatorname{He}_{0} F: \operatorname{ker}\left(d_{0} F\right) & \rightarrow \operatorname{coker}\left(d_{0} F\right)=T_{y} M / \operatorname{Im}\left(d_{0} F\right), \\
v & \mapsto \pi_{\operatorname{coker}\left(d_{0} F\right)}\left(d_{0}^{2} F(v, v)\right),
\end{aligned}
$$

where we denoted by $\pi_{\text {coker }\left(d_{0} F\right)}$ the projection of $T_{y} M$ onto coker $\left(d_{0} F\right)$. Every $\lambda \in \operatorname{Im}\left(d_{0} F\right)^{\perp}$ well-defines a real-valued quadratic form on $\operatorname{ker}\left(d_{0} F\right)$, which is given by (see e.g. [2], Sect. 12.3.1):

$$
\begin{aligned}
\lambda \mathrm{He}_{0} F: \operatorname{ker}\left(d_{0} F\right) & \rightarrow \mathbb{R}, \\
v & \mapsto \int_{0}^{1}\left\langle\lambda,\left[\int_{0}^{t} v_{1}(\tau) X_{1}+v_{2}(\tau) g_{\tau} \mathrm{d} \tau, v_{1}(t) X_{1}+v_{2}(t) g_{t}\right](y)\right\rangle \mathrm{d} t .
\end{aligned}
$$

Since $\lambda \in \operatorname{Im}\left(d_{0} F\right)^{\perp}$, then $\left\langle\lambda, X_{1}(y)\right\rangle=0$ and $\left\langle\lambda, g_{t}(y)\right\rangle \equiv 0$ for every $t \in I$, implying by differentiation that also $\left\langle\lambda,\left[X_{1}, g_{t}\right](y)\right\rangle \equiv 0$ on $I$. Therefore $\lambda \mathrm{He}_{0} F$ reduces to

$$
\lambda \mathrm{He}_{0} F(v)=\int_{0}^{1}\left\langle\lambda,\left[\int_{0}^{t} v_{2}(\tau) g_{\tau} \mathrm{d} \tau, v_{2}(t) g_{t}\right](y)\right\rangle \mathrm{d} t, \quad v \in \operatorname{ker}\left(d_{0} F\right) .
$$

The Hessian leads to a second $L^{2}\left(I, \mathbb{R}^{2}\right)$-orthogonal decomposition of $\operatorname{ker}\left(d_{0} F\right)$ (compare with (2.28)), that is:

$$
\operatorname{ker}\left(d_{0} F\right)=P(\lambda) \oplus N(\lambda) \oplus Z(\lambda)
$$

where $P(\lambda)$ and $N(\lambda)$ are the positive and the negative eigenspace of $\lambda \mathrm{He}_{0} F$, and $Z(\lambda)$ is its kernel. By Theorem 7.1 of [11], this decomposition can be taken orthogonal also with respect to $\lambda \mathrm{He}_{0} F$.

Remark 2.10. Observe that $\lambda \mathrm{He}_{0} F$ does not depend on $v_{1}$. Then, for every $\lambda \in \operatorname{Im}\left(d_{0} F\right)^{\perp}$ the subspace $Z_{1} \subset \operatorname{ker}\left(d_{0} F\right)$, consisting of the controls with zero mean, is contained in $Z(\lambda)$.

Remark 2.11. Since $\gamma$ is strictly singular, we have that $\operatorname{Im}\left(d_{0} \mathcal{F}\right)^{\perp}=\left\{\bar{\lambda}=(\lambda, 0) \mid \lambda \in \operatorname{Im}\left(d_{0} F\right)^{\perp}\right\}$. This implies that, given $\lambda \in \operatorname{Im}\left(d_{0} F\right)^{\perp},(2.33)$ holds also for $\bar{\lambda} \mathrm{He}_{0} \mathcal{F}: \operatorname{ker}\left(d_{0} \mathcal{F}\right) \rightarrow \mathbb{R}$, the only difference being the domain $\operatorname{ker}\left(d_{0} \mathcal{F}\right)$ strictly smaller than $\operatorname{ker}\left(d_{0} F\right)$. 


\section{Modding out the Kernel of the Hessian maP}

The kernel $Z(\lambda)$ of $\lambda \mathrm{He}_{0} F$ contains all the controls of the form $\left(v_{1}, 0\right)$ with $v_{1}$ with zero mean. These elements are troublesome for the construction of a normal form, since the Hessian does not depend at all on them. We introduce a change of coordinates (indeed, a reparametrization of the time) that allows to forget about them in the sequel. This map, however, is only a homeomorphism: the space is too degenerate to allow for a diffeomorphism. Moreover, our construction does not work in $L^{2}\left(I, \mathbb{R}^{2}\right)$.

The point is that in every $L^{2}\left(I, \mathbb{R}^{2}\right)$-neighborhood of $(1,0)$ there are controls $\left(1+v_{1}, 0\right)$ where $1+v_{1}$ does not have a constant sign, which means that the time-reparametrization would not be monotone. To preserve the monotonicity of the time, we must restrict our arguments only to the vector subspace $L^{\infty}(I, \mathbb{R}) \oplus L^{2}(I, \mathbb{R})$.

\subsection{The reparametrization map}

Given $v \in L^{\infty}(I, \mathbb{R})$ we denote by $\bar{v}$ its integral mean, as in the previous section. Every element in $v \in$ $L^{\infty}(I, \mathbb{R})$ decomposes as $\bar{v}+(v-\bar{v})$, and the two summands are orthogonal with respect to the $L^{2}(I, \mathbb{R})$-product.

Given $\alpha>0$, we consider the open set

$$
\mathcal{V}_{2}=\left\{v \in L^{\infty}(I, \mathbb{R}) \oplus L^{2}(I, \mathbb{R}) \mid 1+v_{1}-\bar{v}_{1}>\alpha \text { a.e. on } I\right\}
$$

For $v \in \mathcal{V}_{2}$ we define the time-reparametrization map $\phi_{v}: I \rightarrow I$ by the position:

$$
\phi_{v}(t):=\int_{0}^{t} 1+v_{1}(\tau)-\bar{v}_{1} \mathrm{~d} \tau
$$

Definition 3.1. We define the reparametrization map $\rho: \mathcal{V}_{2} \rightarrow L^{\infty}(I, \mathbb{R}) \oplus L^{2}(I, \mathbb{R})$ by the formula

$$
\rho\left(v_{1}, v_{2}\right)=\left(\bar{v}_{1}+\left(1+\bar{v}_{1}\right)\left(v_{1}-\bar{v}_{1}\right), \dot{\phi}_{v}\left(v_{2} \circ \phi_{v}\right)\right) \text {. }
$$

It is not difficult to compute the inverse map $\rho^{-1}: \rho\left(\mathcal{V}_{2}\right) \rightarrow \mathcal{V}_{2}$ :

$$
\left(v_{1}, v_{2}\right) \mapsto\left(\bar{v}_{1}+\frac{v_{1}-\bar{v}_{1}}{1+\bar{v}_{1}}, \frac{v_{2} \circ \psi_{v}^{-1}}{\dot{\psi}_{v} \circ \psi_{v}^{-1}}\right),
$$

where this time, for $v \in \rho\left(\mathcal{V}_{2}\right)$, the time-reparametrization $\psi_{v}: I \rightarrow I$ is given by

$$
\psi_{v}(t)=\int_{0}^{t} 1+\frac{v_{1}(\tau)-\bar{v}_{1}}{1+\bar{v}_{1}} \mathrm{~d} \tau
$$

Remark 3.2. Since $\psi_{v}(0)=0, \psi_{v}(1)=1$, and $\dot{\psi}_{v}>\alpha>0$ a.e. on $I$, the map $\psi_{v}$ is a strictly monotone bijection of $I$ onto itself. In particular, $\psi_{v}^{-1}$ is absolutely continuous on $I$. Analogous conclusions are valid as well for the $\operatorname{map} \phi_{v}$.

Remark 3.3. If $\mathcal{V}_{2}$ is also contained in the local chart $\mathcal{V}_{1}$ of Definition 2.7 , by the change of variables $t:=\phi_{v}(s)$ we find that:

$$
\begin{aligned}
(F \circ \rho)\left(v_{1}, v_{2}\right) & =x_{0} \odot \overrightarrow{\exp } \int_{0}^{1}\left(1+v_{1}(s)-\bar{v}_{1}\right)\left(1+\bar{v}_{1}\right) X_{1}+\dot{\phi}_{v}(s) v_{2}\left(\phi_{v}(s)\right) X_{2} \mathrm{~d} s \\
& =y \odot \overrightarrow{\exp } \int_{0}^{1} \bar{v}_{1} X_{1}+v_{2}(t) g_{t} \mathrm{~d} t,
\end{aligned}
$$


i.e., $F \circ \rho$ does not depend explicitly on the zero-mean part of $v_{1}$ anymore. The passage from the first to the second line holds by the change of variables $t=\phi_{v}(s)$, and noticing that $\dot{\phi}_{v}(s)=1+v_{1}(s)-\bar{v}_{1}$.

\subsection{Regularity properties of $\rho$}

We prove in this section that $\rho$ is an homeomorphism onto its image.

Lemma 3.4. Let $v \in \rho\left(\mathcal{V}_{2}\right)$, and $\left(v_{n}\right)_{n \in \mathbb{N}}$ be a sequence converging to $v$ in $L^{\infty}(I, \mathbb{R}) \oplus L^{2}(I, \mathbb{R})$. Then:

(i) $\psi_{v_{n}} \rightarrow \psi_{v}$ uniformly on $I$;

(ii) $\psi_{v_{n}}^{-1} \rightarrow \psi_{v}^{-1}$ pointwise on $I$.

Proof. The proof of (i) is trivial and left as an exercise. We pass to (ii). Let $s \in I$ and let us define $t:=\psi_{v}^{-1}(s)$, $t_{n}:=\psi_{v_{n}}^{-1}(s)$. Let $\lim _{n \rightarrow \infty} t_{n}:=\bar{t}$. Assume, by contradiction, that $\bar{t}>t$. By the triangular inequality, for every $\varepsilon>0$ there exists $n_{\varepsilon} \in \mathbb{N}$ such that for every $n \geq n_{\varepsilon}$ one has

$$
s=\psi_{v}(t)<\psi_{v}(\bar{t}) \leq \psi_{v_{n}}\left(t_{n}\right)+3 \varepsilon=s+3 \varepsilon,
$$

where the first strict inequality holds since $\psi_{v}$ is strictly monotone. By the arbitrariness of $\varepsilon$, we conclude that $s<s$, obtaining a contradiction. The case $\bar{t}<t$ is similar: indeed for every $\varepsilon>0$ there exists in this case $n_{\varepsilon} \in \mathbb{N}$, such that for every $n \geq n_{\varepsilon}$ we have

$$
s=\psi_{v}(t)>\psi_{v}(\bar{t}) \geq \psi_{v_{n}}\left(t_{n}\right)-3 \varepsilon=s-3 \varepsilon .
$$

We deduce that $s>s$, obtaining once again an absurd.

Proposition 3.5. The map $\rho: \mathcal{V}_{2} \rightarrow \rho\left(\mathcal{V}_{2}\right)$ is an homeomorphism.

Proof. We only prove the continuity of $\rho^{-1}$, since the continuity of $\rho$ follows by similar arguments.

Let $v \in \rho\left(\mathcal{V}_{2}\right)$ and let $\left(v_{n}\right)_{n \in \mathbb{N}}$ be a sequence converging to $v$ in $L^{\infty}(I, \mathbb{R}) \oplus L^{2}(I, \mathbb{R})$. We need to show that

$$
\lim _{n \rightarrow \infty}\left(\bar{v}_{n, 1}+\frac{\left(v_{n, 1}-\bar{v}_{n, 1}\right)}{1+\bar{v}_{n, 1}}, \frac{v_{n, 2} \circ \psi_{v_{n}}^{-1}}{\dot{\psi}_{v_{n}} \circ \psi_{v_{n}}^{-1}}\right)=\left(\bar{v}_{1}+\frac{\left(v_{1}-\bar{v}_{1}\right)}{1+\bar{v}_{1}}, \frac{v_{2} \circ \psi_{v}^{-1}}{\dot{\psi}_{v} \circ \psi_{v}^{-1}}\right) \text { in } L^{\infty}(I, \mathbb{R}) \oplus L^{2}(I, \mathbb{R}) .
$$

The convergence of the first factor is clear as $v_{n, 1}$ tends to $v_{1}$ in $L^{\infty}(I, \mathbb{R})$. Moreover, it is also clear that

$$
\lim _{n \rightarrow \infty} \dot{\psi}_{v_{n}}=\dot{\psi}_{v} \text { in } L^{\infty}(I, \mathbb{R})
$$

in particular, $\dot{\psi}_{v_{n}}>\alpha$ a.e. on $I$ for $n$ sufficiently large. Then it suffices to prove that

$$
\lim _{n \rightarrow \infty} \int_{0}^{1}\left|\frac{\left(v_{n, 2} \circ \psi_{v_{n}}^{-1}\right)(s)}{\left(\dot{\psi}_{v_{n}} \circ \psi_{v_{n}}^{-1}\right)(s)}-\frac{\left(v_{2} \circ \psi_{v}^{-1}\right)(s)}{\left(\dot{\psi}_{v} \circ \psi_{v}^{-1}\right)(s)}\right|^{2} \mathrm{~d} s=0 .
$$


We bound (3.8) in two steps. We begin with:

$$
\begin{aligned}
& \int_{0}^{1}\left|\frac{\left(v_{n, 2} \circ \psi_{v_{n}}^{-1}\right)(s)}{\left(\dot{\psi}_{v_{n}} \circ \psi_{v_{n}}^{-1}\right)(s)}-\frac{\left(v_{2} \circ \psi_{v}^{-1}\right)(s)}{\left(\dot{\psi}_{v} \circ \psi_{v}^{-1}\right)(s)}\right|^{2} \mathrm{~d} s \\
\leq & 2 \int_{0}^{1}\left|\frac{\left(v_{n, 2} \circ \psi_{v_{n}}^{-1}\right)(s)}{\left(\dot{\psi}_{v_{n}} \circ \psi_{v_{n}}^{-1}\right)(s)}-\frac{\left(v_{2} \circ \psi_{v_{n}}^{-1}\right)(s)}{\left(\dot{\psi}_{v} \circ \psi_{v_{n}}^{-1}\right)(s)}\right|^{2} \mathrm{~d} s+2 \int_{0}^{1}\left|\frac{\left(v_{2} \circ \psi_{v_{n}}^{-1}\right)(s)}{\left.\mid \dot{\psi}_{v} \circ \psi_{v_{n}}^{-1}\right)(s)}-\frac{\left(v_{2} \circ \psi_{v}^{-1}\right)(s)}{\left(\dot{\psi}_{v} \circ \psi_{v}^{-1}\right)(s)}\right|^{2} \mathrm{~d} s .
\end{aligned}
$$

After the change of variables $t:=\psi_{v_{n}}^{-1}(s)$, by the Hölder inequality we find that for $n$ large enough the first term admits the upper bound

$$
\frac{4}{\alpha}\left\|v_{n, 2}-v_{2}\right\|_{L^{2}(I, \mathbb{R})}^{2}+\frac{4}{\alpha^{3}}\left\|\dot{\psi}_{v_{n}}-\dot{\psi}_{v}\right\|_{L^{\infty}(I, \mathbb{R})}^{2}\left\|v_{2}\right\|_{L^{2}(I, \mathbb{R})}^{2},
$$

therefore it converges to zero as $n \rightarrow \infty$.

Expanding the square and applying the obvious changes of variables, we see that the second term in (3.9) is equal to

$$
2 \int_{0}^{1} \frac{v_{2}(t)^{2}}{\dot{\psi}_{v}(t)^{2}} \dot{\psi}_{v_{n}}(t) \mathrm{d} t+2 \int_{0}^{1} \frac{v_{2}(t)^{2}}{\dot{\psi}_{v}(t)} \mathrm{d} t-4 \int_{0}^{1} \frac{v_{2}(t)\left(v_{2} \circ \psi_{v}^{-1} \circ \psi_{v_{n}}\right)(t)}{\left(\dot{\psi}_{v} \circ \psi_{v}^{-1} \circ \psi_{v_{n}}\right)(t)} \frac{\dot{\psi}_{v_{n}}(t)}{\dot{\psi}_{v}(t)} \mathrm{d} t .
$$

By the Hölder inequality we have

$$
\lim _{n \rightarrow \infty} \int_{0}^{1}\left|\frac{v_{2}(t)^{2}}{\dot{\psi}_{v}(t)^{2}} \dot{\psi}_{v_{n}}(t)-\frac{v_{2}(t)^{2}}{\dot{\psi}_{v}(t)}\right| \mathrm{d} t \leq \frac{\left\|v_{2}\right\|_{L^{2}(I, \mathbb{R})}^{2}}{\alpha^{2}} \lim _{n \rightarrow \infty}\left\|\dot{\psi}_{v_{n}}-\dot{\psi}_{v}\right\|_{L^{\infty}(I, \mathbb{R})}=0 .
$$

Next, we claim that

$$
\lim _{n \rightarrow \infty} \int_{0}^{1} \frac{v_{2}(t)\left(v_{2} \circ \psi_{v}^{-1} \circ \psi_{v_{n}}\right)(t)}{\left(\dot{\psi}_{v} \circ \psi_{v}^{-1} \circ \psi_{v_{n}}\right)(t)} \frac{\dot{\psi}_{v_{n}}(t)}{\dot{\psi}_{v}(t)} \mathrm{d} t=\int_{0}^{1} \frac{v_{2}(t)^{2}}{\dot{\psi}_{v}(t)} \mathrm{d} t
$$

The proof of this last limit is made in three steps. First, since $\dot{\psi}_{v_{n}}$ converges to $\dot{\psi}_{v}$ in $L^{\infty}(I, \mathbb{R})$, it is sufficient to show that

$$
\lim _{n \rightarrow \infty} \int_{0}^{1} \frac{v_{2}(t)\left(v_{2} \circ \psi_{v}^{-1} \circ \psi_{v_{n}}\right)(t)}{\left(\dot{\psi}_{v} \circ \psi_{v}^{-1} \circ \psi_{v_{n}}\right)(t)} \mathrm{d} t=\int_{0}^{1} \frac{v_{2}(t)^{2}}{\dot{\psi}_{v}(t)} \mathrm{d} t
$$

given $\varepsilon>0$ we can always assume to work with $n$ bigger than some $n_{\varepsilon}>\mathbb{N}$ so that

$$
\left|\frac{\dot{\psi}_{v_{n}}(t)}{\dot{\psi}_{v}(t)}-1\right|<\varepsilon
$$

almost everywhere on $I$.

Next, given $\varepsilon>0$, we choose $g \in C^{\infty}(I, \mathbb{R})$ such that $\left\|g-v_{2}\right\|_{L^{2}(I, \mathbb{R})} \leq \varepsilon$ and $n_{\varepsilon} \in \mathbb{N}$ such that $\| g-g \circ \psi_{v}^{-1} \circ$ $\psi_{v_{n}} \|_{L^{\infty}(I, \mathbb{R})} \leq \varepsilon$ for every $n \geq n_{\varepsilon}$. This is possible because $\psi_{v_{n}}$ converges uniformly to $\psi_{v}$ on $I$, and $g \circ \psi_{v}^{-1}$ is 
uniformly continuous on the interval. By the Cauchy-Schwartz inequality and the triangular inequality we find that

$$
\begin{aligned}
& \int_{0}^{1}\left|\frac{v_{2}(t)\left(v_{2} \circ \psi_{v}^{-1} \circ \psi_{v_{n}}\right)(t)-v_{2}(t)^{2}}{\left(\dot{\psi}_{v} \circ \psi_{v}^{-1} \circ \psi_{v_{n}}\right)(t)}\right| \mathrm{d} t \leq \frac{\left\|v_{2}\right\|_{L^{2}(I, \mathbb{R})}}{\alpha} \cdot\left(\left\|g-v_{2}\right\|_{L^{2}(I, \mathbb{R})}\right. \\
& \left.\quad+\left\|g-g \circ \psi_{v}^{-1} \circ \psi_{v_{n}}\right\|_{L^{2}(I, \mathbb{R})}+\left\|g \circ \psi_{v}^{-1} \circ \psi_{v_{n}}-v_{2} \circ \psi_{v}^{-1} \circ \psi_{v_{n}}\right\|_{L^{2}(I, \mathbb{R})}\right) \leq \frac{3 \varepsilon\left\|v_{2}\right\|_{L^{2}(I, \mathbb{R})}}{\alpha} .
\end{aligned}
$$

It remains to show that

$$
\lim _{n \rightarrow \infty} \int_{0}^{1} \underbrace{\left|\frac{v_{2}(t)^{2}}{\left(\dot{\psi}_{v} \circ \psi_{v}^{-1} \circ \psi_{v_{n}}\right)(t)}-\frac{v_{2}(t)^{2}}{\dot{\psi}_{v}(t)}\right|}_{:=f_{n}(t)} \mathrm{d} t=0 .
$$

However, we have that $f_{n} \rightarrow 0$ pointwise on $I$ by Lemma 3.4; moreover for $n$ large enough there holds the estimate $f_{n}(t) \leq \frac{2 v_{2}(t)^{2}}{\alpha}$, and therefore (3.13) follows by the dominated convergence theorem. We deduce (3.11) combining (3.12) and (3.13).

\section{Conjugate Points AlONG CORANK-ONE REgular STRICTLY Singular CURVES}

We start in this section a closer study of the map $F \circ \rho$ on the open set $\mathcal{V}_{2} \subset L^{\infty}(I, \mathbb{R}) \oplus L^{2}(I, \mathbb{R})$. As we previously shown, $F \circ \rho$ depends only on $\bar{v}_{1}:=\int_{0}^{1} v_{1}(t) \mathrm{d} t \in \mathbb{R}$ and $v_{2}$. We focus on the restricted maps

$$
\mathrm{F}:=\left.F \circ \rho\right|_{\mathbb{R} \oplus L^{2}(I, \mathbb{R})} \text { and } \mathrm{J}:=\left.J \circ \rho\right|_{\mathbb{R} \oplus L^{2}(I, \mathbb{R})} ;
$$

the first-order analysis for $\mathrm{F}$ and $\mathrm{J}$, and the second-order expansion of $\mathrm{F}$ are formally the same as those for $F$ and $J$. In particular, $\operatorname{Im}\left(d_{0} \mathrm{~F}\right)$ has codimension one in $T_{y} M$, and coincides with $\operatorname{Im}\left(d_{0} F\right)$ by $(2.27)$.

\subsection{Conjugate points}

Fix a corank-one regular strictly singular curve $\gamma$ and assume that

$$
\operatorname{Im}\left(d_{0}(\mathrm{~F}, \mathrm{~J})\right)^{\perp}=\mathbb{R} \cdot(\lambda, 0), \quad \lambda \in \operatorname{Im}\left(d_{0} \mathrm{~F}\right)^{\perp} .
$$

Thus $\gamma$ admits (up to real multiples) a unique extremal lift $\eta$, which is necessarily abnormal. We return to the expression of $\mathrm{F}(v)$, this time interpreting it as a perturbation of the autonomous flow $e^{\int_{0}^{1} v_{2}(t) \mathrm{d} t X_{2}}$. Define $w_{2}(t):=\int_{0}^{t} v_{2}(\tau) \mathrm{d} \tau$. By $(2.9)$ we obtain:

$$
\begin{aligned}
\mathrm{F}(v) & =x_{0} \odot \overrightarrow{\exp } \int_{0}^{1}\left(1+\bar{v}_{1}\right) e_{*}^{-w_{2}(t) X_{2}} X_{1} \mathrm{~d} t \odot e^{w_{2}(1) X_{2}} \\
& =y \odot \overrightarrow{\exp } \int_{0}^{1}\left(1+\bar{v}_{1}\right) e_{*}^{-w_{2}(t) g_{t}} X_{1}-X_{1} \mathrm{~d} t \odot e^{w_{2}(1) X_{2}},
\end{aligned}
$$

therefore $\mathrm{F}$ can be thought as a map depending on $\left(\bar{v}_{1}, w_{2}(1), w_{2}(t)\right) \in \mathbb{R}^{2} \oplus L^{2}(I, \mathbb{R})$. Let us detail a bit more this construction. 
The smooth immersion

$$
\begin{aligned}
\phi: L^{2}(I, \mathbb{R}) & \rightarrow \mathbb{R} \oplus L^{2}(I, \mathbb{R}) \\
v_{2} & \mapsto\left(w_{2}(1), w_{2}(t)\right)
\end{aligned}
$$

allows to identify $L^{2}(I, \mathbb{R})$ with a vector subspace of $\mathbb{R} \oplus L^{2}(I, \mathbb{R})$ (endowed with its standard norm $)^{5}$; furthermore $\mathrm{F}$ can be extended naturally to a smooth map $\stackrel{\circ}{\mathrm{F}}$ on $\mathbb{R}^{2} \oplus L^{2}(I, \mathbb{R})$ by the formula

$$
\stackrel{\circ}{\mathrm{F}} \circ \Phi=\mathrm{F},
$$

where $\Phi=(\operatorname{Id}, \phi)$ is an immersion of $\mathbb{R} \oplus L^{2}(I, \mathbb{R})$ into $\mathbb{R}^{2} \oplus L^{2}(I, \mathbb{R})$, and $\stackrel{\circ}{\mathrm{F}}$ is given by

$$
\stackrel{\circ}{\mathrm{F}}(v, c, w):=y \odot \overrightarrow{\exp } \int_{0}^{1}(1+v) e_{*}^{-w(t) g_{t}} X_{1}-X_{1} \mathrm{~d} t \odot e^{c X_{2}}
$$

Expanding (4.5), it is not difficult to see that

$$
d_{0} \stackrel{\circ}{\mathrm{F}}(v, c, w)=v X_{1}(y)+c X_{2}(y)-\int_{0}^{1} w(t) \dot{g}_{t}(y) \mathrm{d} t
$$

then we can find a constant $C>0$ such that

$$
|v| \leq C\|w\|_{L^{2}(I, \mathbb{R})}, \quad|c| \leq C\|w\|_{L^{2}(I, \mathbb{R})},
$$

for every $(v, c, w) \in \operatorname{ker}\left(d_{0} \stackrel{\circ}{\mathrm{F}}\right)$. Recalling the symmetrisation identity for non-autonomous vector fields on $M$ (see e.g. [2], Lem. 8.30):

$$
\int_{0}^{1} \int_{0}^{t} X_{\tau} \odot X_{t} \mathrm{~d} \tau \mathrm{d} t=\frac{1}{2} \int_{0}^{1}\left[\int_{0}^{t} X_{\tau} \mathrm{d} \tau, X_{t}\right] \mathrm{d} t+\frac{1}{2}\left(\int_{0}^{1} X_{t} \mathrm{~d} t\right) \odot\left(\int_{0}^{1} X_{t} \mathrm{~d} t\right)
$$

and the equality:

$$
y \odot\left(v X_{1}+c X_{2}-\int_{0}^{1} w(t) \dot{g}_{t} \mathrm{~d} t\right) \odot\left(v X_{1}+c X_{2}-\int_{0}^{1} w(t) \dot{g}_{t} \mathrm{~d} t\right)=0,
$$

for every $(v, c, w) \in \operatorname{ker}\left(d_{0} \stackrel{\circ}{\mathrm{F}}\right)$, with a bit more work we find that:

$$
\lambda \operatorname{He}_{0} \stackrel{\circ}{\mathrm{F}}(v, c, w)=\int_{0}^{1}\left\langle\lambda,\left[\dot{g}_{t}, g_{t}\right](y)\right\rangle w(t)^{2} \mathrm{~d} t+\int_{0}^{1}\left\langle\lambda,\left[c X_{2}+\int_{0}^{t} w(\tau) \dot{g}_{\tau} \mathrm{d} \tau, w(t) \dot{g}_{t}\right](y)\right\rangle \mathrm{d} t
$$

for every $\lambda \in \operatorname{Im}\left(d_{0} \stackrel{\circ}{\mathrm{F}}\right)^{\perp}$.

Remark 4.1. The operation leading (4.8) is also called a Goh transformation in the literature. We will show in the next section that the first summand is coercive, while the linear operator associated with the second is compact.

In the original $\mathbb{R} \oplus L^{2}(I, \mathbb{R})$ space of controls, the Legendre condition (4.10) is not apparent, and the Hessian cannot be decomposed in this way. This is the essential reason for introducing $\stackrel{\circ}{\mathrm{F}}$, and working in the completion space.

\footnotetext{
${ }^{5}$ Observe that while the inequality $\left\|\phi\left(v_{2}\right)\right\|_{\mathbb{R} \oplus L^{2}(I, \mathbb{R})} \leq 2\left\|v_{2}\right\|_{L^{2}(I, \mathbb{R})}$ is trivial, the two norms are not equivalent.
} 
As it was done in (2.34), we decompose

$$
\operatorname{ker}\left(d_{0} \stackrel{\circ}{\mathrm{F}}\right)=\stackrel{\circ}{\mathrm{Z}} \oplus \stackrel{\circ}{P} \oplus \stackrel{\circ}{N},
$$

respectively kernel, positive and negative eigenspace of $\lambda \mathrm{He}_{0} \stackrel{\circ}{\mathrm{F}}$. As before, this decomposition will be assumed both $\mathbb{R}^{2} \oplus L^{2}(I, \mathbb{R})$ and $\lambda \mathrm{He}_{0} \stackrel{\circ}{\mathrm{F}}$-orthogonal.

Definition 4.2 (Conjugate points). Let $\gamma$ be a corank-one regular strictly singular curve. We say that $y=\gamma(1)$ is a conjugate point along $\gamma$ if $\stackrel{\circ}{Z} \neq\{0\}$. We say that $y$ has multiplicity $k \in \mathbb{N}$ if $\stackrel{Z}{Z}$ has dimension $k$.

Remark 4.3. We can define conjugate points without introducing the reparametrization map, and working directly with the smooth extension $\stackrel{\circ}{F}$ of the endpoint map $F$ on $L^{\infty}(I, \mathbb{R}) \oplus \mathbb{R} \oplus L^{2}(I, \mathbb{R})$. In this case, $\check{Z}^{\circ}$ contains unavoidably the subspace

$$
\check{Z}_{1}:=\left\{(v, 0,0) \mid v \in L^{\infty}(I, \mathbb{R}), \int_{0}^{1} v(t) \mathrm{d} t=0\right\}
$$

and we say that $y=\gamma(1)$ is not conjugate along $\gamma$ if and only if $\stackrel{Z}{Z}=\check{Z}_{1}$, i.e., if and only if $\stackrel{Z}{Z}$ is in some sense "minimal". The multiplicity of a conjugate point coincides in this setting with the codimension of $\dot{Z}_{1}$ in $\dot{Z}^{\circ}$.

\subsection{The Hessian map of a rank-two-nice singular curve}

Assume $y$ to be not conjugate along $\gamma$, i.e., that $\gamma$ is rank-two-nice according to Definition 1.2. Then

$$
\stackrel{\circ}{Z}=\{0\} .
$$

Proposition 4.4 (Legendre's condition). Up to changing the sign of $\lambda$, there exists a constant $\kappa>0$ such that

$$
\left\langle\lambda,\left[\dot{g}_{t}, g_{t}\right](y)\right\rangle \geq \kappa
$$

holds for every $t \in I$.

Proof. Assume by contradiction that (4.10) is zero for some $t_{0} \in I$. Consider the abnormal lift $\lambda: I \rightarrow \Delta^{\perp}$ of $\gamma$, and recall that for every $t \in I$ we have

$$
\lambda(t)=\left(e^{(1-t) X_{1}}\right)^{*} \lambda \in \Delta_{\gamma(t)}^{\perp} \subset T_{\gamma(t)}^{*} M .
$$

If $\left\langle\lambda,\left[g_{t_{0}}, \dot{g}_{t_{0}}\right](y)\right\rangle=0$, then

$$
\left\langle\lambda,\left[e_{*}^{\left(1-t_{0}\right) X_{1}}\left[X_{1}, X_{2}\right], e_{*}^{\left(1-t_{0}\right) X_{1}} X_{2}\right](y)\right\rangle=\left\langle\lambda\left(t_{0}\right),\left[\left[X_{1}, X_{2}\right], X_{2}\right]\left(\gamma\left(t_{0}\right)\right)\right\rangle=0 .
$$

On the other hand, $\lambda \perp \operatorname{Im}\left(d_{0} \stackrel{\circ}{\mathrm{F}}\right)$ gives $\left\langle\lambda,\left[X_{1}, \dot{g}_{t}\right](y)\right\rangle=0$ for every $t \in I$, yielding as well the identity

$$
\left\langle\lambda,\left[X_{1}, \ddot{g}_{t_{0}}\right](y)\right\rangle=\left\langle\lambda\left(t_{0}\right),\left[X_{1},\left[X_{1}, X_{2}\right]\right]\left(\gamma\left(t_{0}\right)\right)\right\rangle=0 .
$$

Were both (4.12) and (4.13) true, we would argue that $\lambda\left(t_{0}\right) \in\left(\Delta_{\gamma\left(t_{0}\right)}^{3}\right)^{\perp}$, contradicting the fact that $\gamma$ is regular. It then suffices to set

$$
\kappa:=\min _{t \in I}\left\langle\lambda,\left[\dot{g}_{t}, g_{t}\right](y)\right\rangle>0
$$

to conclude. 
By (4.8) it is convenient to split $\lambda \mathrm{He}_{0} \stackrel{\circ}{\mathrm{F}}$ as the sum of two quadratic forms, namely:

$$
\begin{aligned}
R: \operatorname{ker}\left(d_{0} \stackrel{\circ}{\mathrm{F}}\right) & \rightarrow \mathbb{R} \\
(v, c, w) & \mapsto \int_{0}^{1}\left\langle\lambda,\left[\dot{g}_{t}, g_{t}\right](y)\right\rangle w(t)^{2} \mathrm{~d} t \\
T: \operatorname{ker}\left(d_{0} \stackrel{\circ}{\mathrm{F}}\right) & \rightarrow \mathbb{R} \\
(v, c, w) & \mapsto \int_{0}^{1}\left\langle\lambda,\left[c X_{2}+\int_{0}^{t} w(\tau) \dot{g}_{\tau} \mathrm{d} \tau, w(t) \dot{g}_{t}\right](y)\right\rangle \mathrm{d} t
\end{aligned}
$$

For every two non-autonomous vector fields $X_{t}, Y_{t}$ there holds the equality

$$
\int_{0}^{1}\left\langle\lambda,\left[\int_{0}^{t} X_{\tau} \mathrm{d} \tau, Y_{t}\right](y)\right\rangle \mathrm{d} t=\int_{0}^{1}\left\langle\lambda,\left[X_{t}, \int_{t}^{1} Y_{\tau} \mathrm{d} \tau\right](y)\right\rangle \mathrm{d} t
$$

then we compute the linear operator associated with $R$ and $T$ by the formula:

$$
\begin{aligned}
(v, c, w) & \mapsto \pi_{\operatorname{ker}\left(d_{0} \stackrel{\circ}{\mathrm{F}}\right)}\left(R^{L}(v, c, w)\right), \\
(v, c, w) & \mapsto \pi_{\operatorname{ker}\left(d_{0}^{\circ} \stackrel{\circ}{\mathrm{F}}\right)}\left(T^{L}(v, c, w)\right),
\end{aligned}
$$

where $\pi_{\operatorname{ker}\left(d_{0} \stackrel{\circ}{\mathrm{F}}\right)}$ is the orthogonal projection of $\mathbb{R}^{2} \oplus L^{2}(I, \mathbb{R})$ onto $\operatorname{ker}\left(d_{0} \stackrel{\circ}{\mathrm{F}}\right)$, and $R^{L}: \operatorname{ker}\left(d_{0} \stackrel{\circ}{\mathrm{F}}\right) \rightarrow \mathbb{R}^{2} \oplus L^{2}(I, \mathbb{R})$ and $T^{L}: \operatorname{ker}\left(d_{0} \stackrel{\circ}{\mathrm{F}}\right) \rightarrow \mathbb{R}^{2} \oplus L^{2}(I, \mathbb{R})$ are given, respectively, by:

$$
\begin{aligned}
& R^{L}: \quad(v, c, w) \mapsto\left(\begin{array}{c}
0 \\
0 \\
\left\langle\lambda,\left[\dot{g}_{t}, g_{t}\right](y)\right\rangle w(t)
\end{array}\right) \\
& T^{L}: \quad(v, c, w) \mapsto\left(\begin{array}{c}
0 \\
\frac{1}{2} \int_{0}^{1}\left\langle\lambda,\left[X_{2}, \dot{g}_{t}\right](y)\right\rangle w(t) \mathrm{d} t \\
\frac{1}{2}\left\langle\lambda, c\left[X_{2}, \dot{g}_{t}\right](y)+\left[\int_{0}^{t} w(\tau) \dot{g}_{\tau} \mathrm{d} \tau, \dot{g}_{t}\right](y)-\left[\int_{t}^{1} w(\tau) \dot{g}_{\tau} \mathrm{d} \tau, \dot{g}_{t}\right](y)\right\rangle
\end{array}\right) .
\end{aligned}
$$

Finally, we define the linear operators $\widetilde{L}, \widetilde{R}^{L}$, and $\widetilde{T}^{L}$ on $\mathbb{R}^{2} \oplus L^{2}(I, \mathbb{R})=\operatorname{ker}\left(d_{0} \stackrel{\circ}{\mathrm{F}}\right) \oplus \stackrel{\circ}{E}$ by the positions:

$$
\widetilde{R}^{L}=\left(R^{L}, 0\right), \quad \widetilde{T}^{L}=\left(T^{L}, 0\right), \text { and } \widetilde{L}=\widetilde{R}^{L}+\widetilde{T}^{L} .
$$

Proposition 4.5. $\widetilde{T}^{L}$ is compact and self-adjoint. In particular, $\widetilde{L}$ is self-adjoint.

Proof. The self-adjointness of $\widetilde{T}^{L}$ follows directly from the same property for $T^{L}$ :

$$
\begin{aligned}
& \left(T^{L}\left(v_{1}, c_{1}, w_{1}\right),\left(v_{2}, c_{2}, w_{2}\right)\right) \\
& \quad=\frac{1}{2}\left(\int_{0}^{1}\left\langle\lambda,\left[c_{2} X_{2}, \dot{g}_{t} w_{1}(t)\right](y)\right\rangle \mathrm{d} t+\int_{0}^{1}\left\langle\lambda,\left[c_{1} X_{2}, \dot{g}_{t} w_{2}(t)\right](y)\right\rangle \mathrm{d} t\right) \\
& \quad+\frac{1}{2} \int_{0}^{1}\left\langle\lambda,\left[\int_{0}^{t} w_{1}(\tau) \dot{g}_{\tau} \mathrm{d} \tau, \dot{g}_{t} w_{2}(t)\right](y)\right\rangle \mathrm{d} t-\frac{1}{2} \int_{0}^{1}\left\langle\lambda,\left[\int_{t}^{1} w_{1}(\tau) \dot{g}_{\tau} \mathrm{d} \tau, \dot{g}_{t} w_{2}(t)\right](y)\right\rangle \mathrm{d} t \\
& \quad=\left(\left(v_{1}, c_{1}, w_{1}\right), T^{L}\left(v_{2}, c_{2}, w_{2}\right)\right)
\end{aligned}
$$

for every $\left(v_{1}, c_{1}, w_{1}\right)$ and $\left(v_{2}, c_{2}, w_{2}\right) \in \operatorname{ker}\left(d_{0} \stackrel{\circ}{\mathrm{F}}\right)$, where in the last line we used $(4.15)$ to reverse the role of $w_{1}$ and $w_{2}$. 
Similarly, the compactness of $\widetilde{T}^{L}$ is a consequence of the compactness of $T^{L}$. Defining

$$
K(t, \tau):=\left\langle\lambda,\left[\dot{g}_{\tau}, \dot{g}_{t}\right](y)\right\rangle \chi_{[0, t]}(\tau)-\left\langle\lambda,\left[\dot{g}_{\tau}, \dot{g}_{t}\right](y)\right\rangle \chi_{[t, 1]}(\tau), \quad K(t, \tau) \in L^{2}\left(I^{2}, \mathbb{R}\right)
$$

it suffices to show that

$$
(v, c, w) \mapsto c\left\langle\lambda,\left[X_{2}, g_{t}\right](y)\right\rangle+\int_{0}^{1} K(t, \tau) w(\tau) \mathrm{d} \tau
$$

is compact. In fact, $(v, c, w) \mapsto c\left\langle\lambda,\left[X_{2}, \dot{g}_{t}\right](y)\right\rangle$ is a rank-one operator, while the compactness of $(v, c, w) \mapsto$ $\int_{0}^{1} K(t, \tau) w(\tau) \mathrm{d} \tau$ is classical, and proved e.g. in Chapter 6 of [12]. The result follows.

To see that $\widetilde{L}$ is self-adjoint it suffices instead to show that $\widetilde{R}^{L}$ is symmetric, which is trivial since

$$
\int_{0}^{1}\left(\left\langle\lambda,\left[\dot{g}_{t}, g_{t}\right](y)\right\rangle w_{1}(t)\right) \cdot w_{2}(t) \mathrm{d} t=\int_{0}^{1} w_{1}(t) \cdot\left(\left\langle\lambda,\left[\dot{g}_{t}, g_{t}\right](y)\right\rangle w_{2}(t)\right) \mathrm{d} t
$$

for every $w_{1}, w_{2} \in L^{2}(I, \mathbb{R})$

\subsection{On the coercivity of the Hessian map}

The map $R$ in (4.14) endows $\operatorname{ker}\left(d_{0} \stackrel{\circ}{\mathrm{F}}\right)$ with a norm which is equivalent to the product norm induced from $\mathbb{R}^{2} \oplus L^{2}(I, \mathbb{R})$. In fact, if we define $\ell:=\max _{t \in I}\left\langle\lambda,\left[\dot{g}_{t}, g_{t}\right](y)\right\rangle>0$, then by Proposition 4.4 and formula (4.7) for every $(v, c, w) \in \operatorname{ker}\left(d_{0} \stackrel{\circ}{\mathrm{F}}\right)$ we have

$$
\frac{\kappa}{3\left(C^{2}+1\right)}\|(v, c, w)\|^{2} \leq R(v, c, w) \leq \ell\|(v, c, w)\|^{2} .
$$

Proposition 4.6. There exists a constant $K>0$ such that,

$$
\lambda \mathrm{He}_{0} \stackrel{\circ}{\mathrm{F}}(v, c, w) \geq K\|(v, c, w)\|^{2}
$$

for every $(v, c, w) \in \stackrel{\circ}{P}$.

Proof. Let us define

$$
\begin{aligned}
\alpha: & =\inf \left\{\lambda \operatorname{He}_{0} \stackrel{\circ}{\mathrm{F}}(v, c, w) \mid(v, c, w) \in \stackrel{\circ}{P}, R(v, c, w)=1\right\} \\
& =1+\inf \{T(v, c, w) \mid(v, c, w) \in \stackrel{\circ}{P}, R(v, c, w)=1\} .
\end{aligned}
$$

It suffices to show that $\alpha>0$ to conclude. Indeed, were this true, we would have

$$
\lambda \mathrm{He}_{0} \stackrel{\circ}{\mathrm{F}}(v, c, w) \geq \alpha \frac{\kappa}{3\left(C^{2}+1\right)}\|(v, c, w)\|^{2}
$$

for every $(v, c, w) \in \stackrel{\circ}{P}$, and the proposition would follow with $K:=\alpha \frac{\kappa}{3\left(C^{2}+1\right)}$.

Assume by contradiction that $\alpha=0$. Since the set $\{(v, c, w) \in \stackrel{\circ}{P} \mid R(v, c, w)=1\}$ is norm-bounded in $\mathbb{R}^{2} \oplus$ $L^{2}(I, \mathbb{R})$, by Proposition 4.5 the inf in the second line of $(4.22)$ is actually attained, i.e, there exists $(v, c, w) \in \stackrel{\circ}{P}$ 
such that $R(v, c, w)=1$ for which

$$
\lambda \mathrm{He}_{0} \stackrel{\circ}{\mathrm{F}}(v, c, w)=0,
$$

and we conclude by Lemma 6.2 of [11] that ${ }^{6}$

$$
\lambda \operatorname{He}_{0} \stackrel{\circ}{\mathrm{F}}\left((v, c, w),\left(v^{\prime}, c^{\prime}, w^{\prime}\right)\right)=0
$$

for every $\left(v^{\prime}, c^{\prime}, w^{\prime}\right) \in \stackrel{\circ}{P}$. Since $\stackrel{\circ}{P}$ and $\stackrel{\circ}{N}$ are also orthogonal with respect to $\lambda \mathrm{He}_{0} \stackrel{\circ}{\mathrm{F}}$, we argue that $(v, c, w) \in$ $\stackrel{\circ}{Z}=\{0\}$, contradicting the fact that $R(v, c, w)=1$.

The next statement contains some useful information about the subspaces $\stackrel{\circ}{P}$ and $\stackrel{\circ}{N}$, and collects essentially the results of Theorem 1 of [20]. We define, for a given $s \in I, \gamma_{s}:=\left.\gamma\right|_{[0, s]}$ and

$$
\eta(s):=\left(e^{(1-s) X_{1}}\right)^{*} \lambda \in T_{\gamma(s)}^{*} M .
$$

as in (4.11). The control associated with $\gamma_{s}$ is $u_{s}:=(1,0) \cdot \chi_{[0, s]}(t)$; therefore the first and second order analysis carried up to now hold for $\gamma_{s}$ as well, with the obvious restriction of the controls to the time-interval $[0, s]$. Observe that, for every $s \in I$, the Hessian map $\eta(s) \mathrm{He}_{\gamma_{s}} \stackrel{\circ}{\mathrm{F}}$ induces the orthogonal splitting

$$
\operatorname{ker}\left(d_{\gamma_{s}} \stackrel{\circ}{\mathrm{F}}\right)=\stackrel{\circ}{Z}_{s} \oplus \stackrel{\circ}{P}_{s} \oplus \stackrel{\circ}{N}_{s}
$$

there are obvious inclusions of these subspaces as $s$ increases, extending by zero their elements on $[s, s+\delta]$ for every $\delta>0$.

Proposition 4.7. Let $\gamma$ be a corank-one regular strictly singular curve.

(i) There exists $s_{0} \in I$ such that $\operatorname{ker}\left(d_{\gamma_{s}} \stackrel{\circ}{\mathrm{F}}\right)=\stackrel{\circ}{P}_{s}$ for every $s \leq s_{0}$.

(ii) Conjugate points along $\gamma$ are isolated, and every conjugate point has a finite multiplicity.

(iii) The negative index of $\lambda \mathrm{He}_{0} \stackrel{\circ}{\mathrm{F}}$ equals the sum of the multiplicities of all the conjugate points along $\gamma$. In particular, $N$ is a finite-dimensional subspace.

Remark 4.8. In [20] the conclusions of Proposition 4.7 were drawn for a control system of the form:

$$
\dot{x}(t)=f(x(t), u(t)) \quad t \in I
$$

and were granted by the existence of a positive constant $C$ such that

$$
\left|\frac{\partial^{2}}{\partial u^{2}} f(x(t), u(t)) \cdot v\right| \geq C|v|
$$

for every $t \in I$. In our setting $\frac{\partial^{2}}{\partial u^{2}} f(x(t), u(t)) \equiv 0$; nonetheless the assumption that $\gamma$ is regular permits to bring the Hessian in form (4.8), and repeat the same analysis.

Proposition 4.9. Let $\gamma \in \Omega(y)$ be a rank-two-nice curve, and let $L$ be the linear operator associated with $\lambda \mathrm{He}_{0} \stackrel{\circ}{\mathrm{F}}$. Then $L$ is invertible.

Proof. Observe that $L$ coincides with the restriction onto $\operatorname{ker}\left(d_{0} \stackrel{\circ}{\mathrm{F}}\right)$ of the self-adjoint linear operator $\widetilde{L}$ defined in (4.17). In particular $L$ can be split as the sum of a self-adjoint linear operator $L_{\dot{P}}$ on $\stackrel{\circ}{P}$ and a self-adjoint linear operator $L_{\stackrel{\circ}{\circ}}$ on $\stackrel{\circ}{N}$.

\footnotetext{
${ }^{6} \mathrm{By}$ a slight abuse of the notation, we identify $\lambda \mathrm{He}_{0} \stackrel{\circ}{\mathrm{F}}$ and its associated bilinear map.
} 


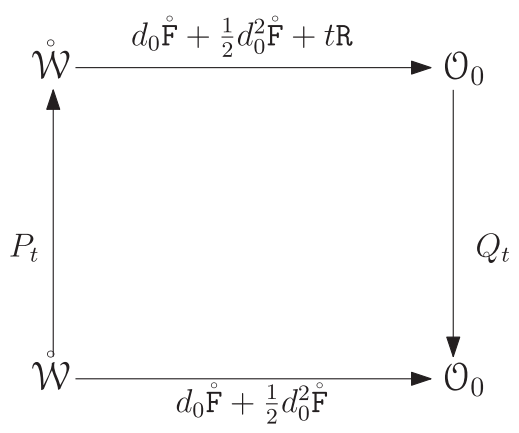

Figure $3 . P_{t}$ and $Q_{t}$ are chosen so that the diagram commutes.

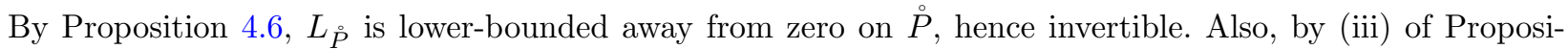
tion 4.7 , we see that $L_{N}$ has trivial kernel on a finite-dimensional space, and is therefore invertible as well. We conclude that $L$ itself is invertible.

\section{Normal Forms AROUND RANK-TWO-NICE SINGUlar CURVES}

Let $\gamma \in \Omega(y)$ be a rank-two-nice curve. In this section we derive a normal form for $\stackrel{\circ}{\mathrm{F}}$ around $\gamma$. We fix coordinates $z:=(v, c, w) \in \mathbb{R}^{2} \oplus L^{2}(I, \mathbb{R})$, a system of local coordinates centered at $y$ in $\mathbb{R}^{m}=\mathbb{R} \oplus \operatorname{Im}\left(d_{0} \stackrel{\circ}{\mathrm{\circ}}\right)$, and we write

$$
\stackrel{\circ}{\mathrm{F}}(z)=d_{0} \stackrel{\circ}{\mathrm{F}}(z)+\frac{1}{2} d_{0}^{2} \stackrel{\circ}{\mathrm{F}}(z)+\mathrm{R}(z)
$$

where $\mathrm{R}$ denotes a remainder term whose first and second derivatives at zero vanish.

Proposition 5.1. There exist neighborhoods of the origin $\mathcal{W} \subset \mathbb{R}^{2} \oplus L^{2}(I, \mathbb{R})$ and $\mathcal{O}_{0} \subset \mathbb{R} \oplus \operatorname{Im}\left(d_{0} \stackrel{\circ}{\mathrm{F}}\right)$, and origin-preserving diffeomorphisms $\sigma: \mathcal{W} \rightarrow \mathcal{W}$ and $\nu: \mathcal{O}_{0} \rightarrow \mathcal{O}_{0}$, such that for every $z \in \mathcal{W}$ there holds the identity:

$$
(\nu \circ \stackrel{\circ}{\mathrm{F}} \circ \sigma)(z)=\left(\lambda \mathrm{He}_{0}(\stackrel{\circ}{\mathrm{F}})\left(z^{\prime}\right), d_{0} \stackrel{\circ}{\mathrm{F}}\left(z^{\prime \prime}\right)\right)
$$

where $z=\left(z^{\prime}, z^{\prime \prime}\right)$ is a coordinate system subordinated to the splitting $\operatorname{ker}\left(d_{0} \stackrel{\circ}{\mathrm{F}}\right) \oplus \stackrel{\circ}{E}$.

Proof. We prove in fact a slightly stronger statement: there exist neighborhoods of the origin $\mathcal{W} \subset \mathbb{R}^{2} \oplus L^{2}(I, \mathbb{R})$ and $\mathcal{O}_{0} \subset \mathbb{R} \oplus \operatorname{Im}\left(d_{0} \stackrel{\circ}{\mathrm{F}}\right)$, such that for every $t \in I$ there exist origin-preserving diffeomorphisms $P_{t}: \mathcal{W} \rightarrow \mathcal{W}$ and $Q_{t}: \mathcal{O}_{0} \rightarrow \mathcal{O}_{0}$, for which the diagram in Figure 3 commutes.

It is then sufficient to set $\sigma:=P_{1}$ and $\nu=Q_{1}$ to prove the proposition.

We construct $P_{t}$ and $Q_{t}$ as the flows of suitable time-dependent vector fields $X_{\tau}$ on $\mathcal{W}$ and $Y_{\tau}$ on $\mathcal{O}_{0}$, i.e. we assume that the families $\left(X_{\tau}\right)_{\tau \in I}$ and $\left(Y_{\tau}\right)_{\tau \in I}$ are locally integrable in time and that $X_{\tau}$ and $Y_{\tau}$ are smooth vector fields for every $\tau \in I$. As in the classical Moser's trick [17] we set

$$
P_{t}:=\overrightarrow{\exp } \int_{0}^{t} X_{\tau} \mathrm{d} \tau \text { and } Q_{t}:=\overleftarrow{\exp } \int_{0}^{t} Y_{\tau} \mathrm{d} \tau
$$

We recall that, in local coordinates, this is equivalent to say that the flows $P_{t}$ and $Q_{t}$ solve, respectively,

$$
\begin{aligned}
\dot{P}_{t}(z) & =X_{t}\left(P_{t}(z)\right) \text { and } \\
\dot{Q}_{t}(z) & =J Q_{t}(z) Y_{t}(z),
\end{aligned}
$$


where by $J Q_{t}(z)$ we denoted the Jacobian of $Q_{t}$ evaluated at $z$. The commutativity condition expressed by the diagram reads

$$
Q_{t} \circ\left(d_{0} \stackrel{\circ}{\mathrm{F}}+\frac{1}{2} d_{0}^{2 \circ} \mathrm{F}+t \mathrm{R}\right) \circ P_{t}(z)=d_{0} \stackrel{\circ}{\mathrm{F}}(z)+\frac{1}{2} d_{0}^{2} \stackrel{\circ}{\mathrm{F}}(z) .
$$

For $t=0$ the identity holds, and upon differentiating with respect to $t$ we obtain:

$$
\begin{aligned}
& J Q\left(\stackrel{\circ}{\mathrm{F}}\left(P_{t}(z)\right)\right) Y_{t}\left(d_{0} \stackrel{\circ}{\mathrm{F}}\left(P_{t}(z)\right)+\frac{1}{2} d_{0}^{2} \stackrel{\circ}{\mathrm{F}}\left(P_{t}(z), P_{t}(z)\right)+t \mathrm{R}\left(P_{t}(z)\right)\right)+J Q\left(\stackrel{\circ}{\mathrm{F}}\left(P_{t}(z)\right)\right) \mathrm{R}\left(P_{t}(z)\right) \\
& \quad+J Q\left(\stackrel{\circ}{\mathrm{F}}\left(P_{t}(z)\right)\right)\left(d_{0} \stackrel{\circ}{\mathrm{F}}\left(X_{t}\left(P_{t}(z)\right)\right)+d_{0}^{2} \stackrel{\circ}{\mathrm{F}}\left(P_{t}(z), X_{t}\left(P_{t}(z)\right)\right)+t d_{P_{t}(z)} \mathrm{R}\left(X_{t}\left(P_{t}(z)\right)\right)\right)=0 .
\end{aligned}
$$

We look for a vector field $Y_{t}$ such that $\pi_{\operatorname{Im}\left(d_{0}{ }^{\circ}\right)}\left(Y_{t}\right)=0$, i.e., of the form $Y_{t}=\left(Y_{t}^{\lambda}, 0\right)$. Taking into account the fact that $P_{t}$ and $Q_{t}$ are diffeomorphisms, the solutions to (5.4) are given as solutions to the following system:

$$
\left\{\begin{array}{l}
d_{0} \stackrel{\circ}{\mathrm{F}} X_{t}(z)+\pi_{\operatorname{Im}\left(d_{0} \stackrel{\circ}{\mathrm{F}}\right)}\left(t d_{z} \mathrm{R}\left(X_{t}(z)\right)+d_{0}^{2} \stackrel{\circ}{\mathrm{F}}\left(z, X_{t}(z)\right)\right)=-\pi_{\operatorname{Im}\left(d_{0} \circ \stackrel{\circ}{\mathrm{F}}\right)} \mathrm{R}(z), \\
Y_{t}^{\lambda}\left(d_{0} \stackrel{\circ}{\mathrm{F}}(z)+\frac{1}{2} d_{0}^{2} \mathrm{\circ}(z, z)+t \mathrm{R}(z)\right)+\left\langle\lambda, d_{0}^{2} \stackrel{\circ}{\mathrm{F}}\left(z, X_{t}(z)\right)\right\rangle+t\left\langle\lambda, d_{z} \mathrm{R} X_{t}(z)\right\rangle=-\langle\lambda, \mathrm{R}(z)\rangle .
\end{array}\right.
$$

Let us fix coordinates $\left(z^{\prime}, z^{\prime \prime}\right)$ on $\mathbb{R}^{2} \oplus L^{2}(I, \mathbb{R})$, subordinated to the $\operatorname{splitting} \operatorname{ker}\left(d_{0} \stackrel{\circ}{\mathrm{F}}\right) \oplus \stackrel{\circ}{E}$. Given $z \in$ $\mathbb{R}^{2} \oplus L^{2}(I, \mathbb{R})$ and $t \in I$, we define the operator $K_{t}(z): \stackrel{\circ}{E} \rightarrow \operatorname{Im}\left(d_{0} \stackrel{\circ}{\mathrm{F}}\right)$ by the formula

$$
K_{t}(z)\left[z^{\prime \prime}\right]=d_{0} \stackrel{\circ}{\mathrm{F}}\left(z^{\prime \prime}\right)+\pi_{\operatorname{Im}\left(d_{0} \stackrel{\circ}{\mathrm{F}}\right)}\left(t d_{z} \mathrm{R}\left(z^{\prime \prime}\right)+d_{0}^{2} \stackrel{\circ}{\mathrm{F}}\left(z, z^{\prime \prime}\right)\right) .
$$

Since $K_{t}(0)=d_{0} \stackrel{\circ}{\mathrm{F}}$ for every $t \in I$, we argue that there exists a neighborhood $\mathcal{W}_{1} \subset \mathbb{R}^{2} \oplus L^{2}(I, \mathbb{R})$ of the origin where, for every $z \in \mathcal{W}_{1}, K_{t}(z)$ is invertible for every $t \in I$.

Then for $z \in \mathcal{W}_{1}$ we write $X_{t}(z)=X_{t}(z)^{\prime}+X_{t}(z)^{\prime \prime}$, and we solve for $X_{t}(z)^{\prime \prime}$ the first equation in (5.5), i.e.

$$
\begin{aligned}
X_{t}(z)^{\prime \prime} & =-K_{t}(z)^{-1}\left[\pi_{\operatorname{Im}\left(d_{0} \stackrel{\circ}{\mathrm{F}}\right)}\left(t d_{z} \mathrm{R}\left(X_{t}(z)^{\prime}\right)+d_{0}^{2} \stackrel{\circ}{\mathrm{F}}\left(z, X_{t}(z)^{\prime}\right)+\mathrm{R}(z)\right)\right] \\
& =-K_{t}(z)^{-1}\left[G_{t}(z)\left(X_{t}(z)^{\prime}\right)\right]
\end{aligned}
$$

where the definition of the map $G_{t}(z): \mathbb{R}^{2} \oplus L^{2}(I, \mathbb{R}) \rightarrow \operatorname{Im}\left(d_{0} \stackrel{\circ}{\mathrm{F}}\right)$ is specified inline between the first and the second line. Observe that $G_{t}(0)$ is the zero map for every $t \in I$.

Next, we substitute $X_{t}(z)^{\prime \prime}$ in the second equation of (5.5), to find $X_{t}(z)^{\prime}$ and $Y_{t}^{\lambda}(z)$ using the second (scalar) equation:

$$
\left(A_{t}[z], X_{t}(z)^{\prime}\right)_{\mathbb{R}^{2} \oplus L^{2}(I, \mathbb{R})}+Y_{t}^{\lambda}\left(d_{0} \stackrel{\circ}{\mathrm{F}}(z)+\frac{1}{2} d_{0}^{2} \stackrel{\circ}{\mathrm{F}}(z, z)+t \mathrm{R}(z)\right)=-\langle\lambda, \mathrm{R}(z)\rangle .
$$

Here, for every $t \in I$, the map $A_{t}: \mathcal{W}_{1} \rightarrow \mathbb{R}^{2} \oplus L^{2}(I, \mathbb{R})$ is defined by duality requiring that

$$
\left(A_{t}[z], x\right)_{\mathbb{R}^{2} \oplus L^{2}(I, \mathbb{R})}:=\left\langle\lambda, d_{0}^{2} \stackrel{\circ}{\mathrm{F}}\left(z, x-K_{t}(z)^{-1}\left[G_{t}(z)(x)\right]\right)+t d_{z} \mathrm{R}\left(x-K_{t}(z)^{-1}\left[G_{t}(z)(x)\right]\right)\right\rangle
$$

for every $x \in \mathbb{R}^{2} \oplus L^{2}(I, \mathbb{R})$.

In particular, the linear map $d_{0} A_{t}: \mathbb{R}^{2} \oplus L^{2}(I, \mathbb{R}) \rightarrow \mathbb{R}^{2} \oplus L^{2}(I, \mathbb{R})$ is defined by the formula

$$
\left(d_{0} A_{t}(z), x\right)_{\mathbb{R}^{2} \oplus L^{2}(I, \mathbb{R})}=\lambda d_{0}^{2} \stackrel{\circ}{\mathrm{F}}(z, x) \text { for every } z, x \in \mathbb{R}^{2} \oplus L^{2}(I, \mathbb{R}),
$$


i.e., we argue that for every $z \in \operatorname{ker}\left(d_{0} \stackrel{\circ}{\mathrm{F}}\right)$ we have

$$
\left(d_{0} A_{t}(z), z\right)_{\mathbb{R}^{2} \oplus L^{2}(I, \mathbb{R})}=\lambda \operatorname{He}_{0} \stackrel{\circ}{\mathrm{F}}(z) .
$$

Stated differently, the linear mapping

$$
\left.\pi_{\operatorname{ker}\left(d_{0} \stackrel{\circ}{\mathrm{F}}\right)} \circ d_{0} A_{t}\right|_{\operatorname{ker}\left(d_{0} \stackrel{\circ}{\mathrm{F}}\right)}
$$

coincides with the linear operator $L: \operatorname{ker}\left(d_{0} \stackrel{\circ}{\mathrm{F}}\right) \rightarrow \operatorname{ker}\left(d_{0} \stackrel{\circ}{\mathrm{F}}\right)$ associated with the Hessian map $\lambda \mathrm{He}_{0} \stackrel{\circ}{\mathrm{F}}$, and therefore it is invertible by Proposition 4.9 .

We denote by $\left(\left.d_{0} \stackrel{\circ}{\mathrm{F}}\right|_{E} ^{\circ}\right)^{*}: T_{0} M \rightarrow \stackrel{\circ}{E}$ the linear operator adjoint to the restriction of $d_{0} \stackrel{\circ}{\mathrm{F}}$ onto $\stackrel{\circ}{E}$, and we consider for $t \in I$ the map

$$
\begin{aligned}
\Psi_{t}: \mathcal{W}_{1} & \rightarrow \mathbb{R}^{2} \oplus L^{2}(I, \mathbb{R}), \\
z & \mapsto \pi_{\operatorname{ker}\left(d_{0} \circ \stackrel{\circ}{\mathrm{F}}\right)} \circ A_{t}(z)+\left(\left.d_{0} \stackrel{\circ}{\mathrm{F}}\right|_{E} ^{\circ}\right)^{*}\left(d_{0} \stackrel{\circ}{\mathrm{F}}(z)+\frac{1}{2} d_{0}^{2} \stackrel{\circ}{\mathrm{F}}(z, z)+t \mathrm{R}(z)\right) .
\end{aligned}
$$

Notice that $\Psi_{t}$ is origin-preserving. Since $d_{0} \Psi_{t}$ is easily seen to be a linear isomorphism of $\mathbb{R}^{2} \oplus L^{2}(I, \mathbb{R})=$ $\operatorname{ker}\left(d_{0} \stackrel{\circ}{\mathrm{F}}\right) \oplus \stackrel{\circ}{E}$ onto itself, we conclude that there exists a neighborhood $\mathcal{W} \subset \mathcal{W}_{1}$ such that $\Psi_{t}$ defines a new system of coordinates on $\mathcal{W}$,

$$
\left(w_{t}(z)^{\prime}, w_{t}(z)^{\prime \prime}\right)=\left(\pi_{\mathrm{ker}\left(d_{0} \circ \circ^{\circ}\right)} \circ A_{t}(z),\left(\left.d_{0} \stackrel{\circ}{\mathrm{F}}\right|_{E} ^{\circ}\right)^{*}\left(d_{0} \stackrel{\circ}{\mathrm{F}}(z)+\frac{1}{2} d_{0}^{2} \stackrel{\circ}{\mathrm{F}}(z, z)+t \mathrm{R}(z)\right)\right)
$$

for every $t \in I$.

Eventually, for every $t \in I$ we find by the Hadamard Lemma a smooth function $\chi_{t}: \mathcal{W} \rightarrow \mathbb{R}^{2} \oplus L^{2}(I, \mathbb{R})$ such that

$$
\begin{aligned}
\langle\lambda, \mathrm{R}(z)\rangle & =\left\langle\lambda,\left(\mathrm{R} \circ \Psi_{t}^{-1}\right)\left(w_{t}(z)^{\prime}, w_{t}(z)^{\prime \prime}\right)\right\rangle \\
& =\left\langle\lambda,\left(\mathrm{R} \circ \Psi_{t}^{-1}\right)\left(0, w_{t}(z)^{\prime \prime}\right)\right\rangle+\left(\left(w_{t}(z)^{\prime}, 0\right), \chi_{t}(z)\right)_{\mathbb{R}^{2} \oplus L^{2}(I, \mathbb{R})},
\end{aligned}
$$

and therefore by comparing this expression with (5.7) we conclude setting

$$
X_{t}(z)^{\prime}:=-\left(\pi_{\operatorname{ker}\left(d_{0} \mathrm{\circ}\right)} \circ \chi_{t}\right)(z) \text { and } Y_{t}^{\lambda}(z):=-\left\langle\lambda,\left(\mathrm{R} \circ \Psi_{t}^{-1} \circ\left(\left.d_{0} \stackrel{\circ}{\mathrm{F}}\right|_{\stackrel{E}{\circ}}\right)^{*}\right)(z)\right\rangle
$$

for every $z \in \mathcal{W}$.

Definition 5.2. With $\stackrel{\circ}{\mathrm{F}}$ as in (4.5), we define the map $\stackrel{\circ}{F}: L^{\infty}(I, \mathbb{R}) \oplus \mathbb{R} \oplus L^{2}(I, \mathbb{R}) \rightarrow M$ by the position

$$
\stackrel{\circ}{F}(v-\bar{v}, \bar{v}, c, w):=\stackrel{\circ}{\mathrm{F}}(\bar{v}, c, w)
$$

Notice that $\stackrel{\circ}{F}$ is indeed an extension of $F \circ \rho$ with respect to the $L^{2}$-factor, and that there is essentially no difference with the extension $\stackrel{\circ}{\mathrm{F}}$ of $\mathrm{F}$, except for the fact that we "forget" the trivial dependence of $F \circ \rho$ on the zero mean part of the $L^{\infty}$-component.

We can now complete the proof of our main result, Theorem 1.3. 
Proof of Theorem 1.3. Let $\pi_{\mathbb{R}^{2} \oplus L^{2}(I, \mathbb{R})}$ denote the projection of $L^{\infty}(I, \mathbb{R}) \oplus \mathbb{R} \oplus L^{2}(I, \mathbb{R})$ onto $\mathbb{R}^{2} \oplus L^{2}(I, \mathbb{R})$. We define $\mathcal{W}:=\pi_{\mathbb{R}^{2} \oplus L^{2}(I, \mathbb{R})}^{-1}(\mathcal{W})$ and the map $\mu: \mathcal{W} \rightarrow \mathcal{W}$ by the formula

$$
\mu(v-\bar{v}, \bar{v}, c, w)=(v-\bar{v}, \sigma(\bar{v}, c, w)),
$$

where $\sigma: \mathcal{W} \rightarrow \mathcal{W}$ is defined as in Proposition 5.1.

We conclude the proof by declaring $\psi$ to be the composition of the origin-preserving map $\nu$ of Proposition 5.1 with the local system of coordinates centered at $y$, and defined at the beginning of this section. Indeed, let $y \in \mathcal{W}$ and let $z:=\pi_{\mathbb{R}^{2} \oplus L^{2}(I, \mathbb{R})}(y) \in \mathcal{W}$. It follows by $(5.11)$ that

$$
\begin{aligned}
\psi \circ \stackrel{\circ}{\mathrm{F}} \circ \mu(y) & =\nu \circ \stackrel{\circ}{\mathrm{F}} \circ \sigma(z) \\
& =\left(\lambda \mathrm{He}_{0}(\stackrel{\circ}{\mathrm{F}})\left(z^{\prime}\right), d_{0} \stackrel{\circ}{\mathrm{F}}\left(z^{\prime \prime}\right)\right) \\
& =\left(\lambda \operatorname{He}_{0}(\stackrel{\circ}{F})\left(y^{\prime}\right), d_{0} \stackrel{\circ}{F}\left(y^{\prime \prime}\right)\right),
\end{aligned}
$$

where $y=y^{\prime}+y^{\prime \prime} \in \operatorname{ker} d_{0} \stackrel{\circ}{F} \oplus \stackrel{\circ}{E}$, and $y^{\prime}$ and $y^{\prime \prime}$ are chosen so that

$$
\pi_{\mathbb{R}^{2} \oplus L^{2}(I, \mathbb{R})}\left(y^{\prime}\right)=z^{\prime}, \quad \pi_{\mathbb{R}^{2} \oplus L^{2}(I, \mathbb{R})}\left(z^{\prime \prime}\right)=y^{\prime \prime} .
$$

Notice that the first and the last equality of this chain are true since $\stackrel{\circ}{F}$ does not depend on the zero-mean component of the $L^{\infty}$-factor of $y$, and also $z^{\prime} \in \operatorname{ker}\left(d_{0} \stackrel{\circ}{\mathrm{F}}\right)$ implies that $y^{\prime} \in \operatorname{ker}\left(d_{0} \stackrel{\circ}{F}\right)$ for every $y^{\prime} \in \pi_{\mathbb{R}^{2} \oplus L^{2}(I, \mathbb{R})}^{-1}\left(z^{\prime}\right)$.

Proof of Proposition 1.4. Let $\left(v_{n}\right)_{n \in \mathbb{N}} \subset \Omega(y)$ be a sequence of singular controls weakly converging to zero in $L^{2}\left(I, \mathbb{R}^{2}\right)$. By Proposition 2.1,

$$
F_{t}\left(v_{n}\right) \rightarrow F_{t}(0) \text { and } d_{v_{n}} F_{t} \rightarrow d_{0} F_{t} \text { uniformly for } t \in I
$$

then we may suppose that $\operatorname{Im}\left(d_{v_{n}} F\right)$ is of codimension one in $T_{y} M$ for every $n \in \mathbb{N}$.

Given $v \in L^{2}\left(I, \mathbb{R}^{2}\right)$ and $t \in I$, we consider the diffeomorphism

$$
\begin{aligned}
P_{0}^{t, v}: & M \rightarrow M \\
x & \mapsto x \odot \overrightarrow{\exp } \int_{0}^{t}\left(1+v_{1}(\tau)\right) X_{1}+v_{2}(\tau) X_{2} \mathrm{~d} \tau
\end{aligned}
$$

and we write $P_{t}^{0, v}$ for $\left(P_{0}^{t, v}\right)^{-1}$. Let us fix a norm $\|\cdot\|$ on $\mathbb{R}^{m}$, and let us take norm-one covectors $\lambda_{v_{n}} \in$ $\operatorname{Im}\left(d_{v_{n}} F\right)^{\perp}$ for every $n \in \mathbb{N}$ and $\lambda_{v} \in \operatorname{Im}\left(d_{v} F\right)^{\perp}$, i.e. $\left\|\lambda_{v_{n}}\right\|=\left\|\lambda_{v}\right\|=1$.

We define

$$
\lambda_{v_{n}}^{0}:=\left(P_{0}^{1, v_{n}}\right)^{*} \lambda_{v_{n}}, \quad \lambda_{v}^{0}:=\left(P_{0}^{1, v}\right)^{*} \lambda_{v}
$$

Since $\lambda_{v_{n}}$ converges to $\lambda_{v}$ and $\left(P_{0}^{1, v_{n}}\right)^{*}$ converges to $\left(P_{0}^{1, v}\right)^{*}$, by construction $\lambda_{v_{n}}^{0} \rightarrow \lambda_{v}^{0}$. Similarly, we argue that $\lambda_{v_{n}}(t):=\left(P_{t}^{0, v_{n}}\right)^{*} \lambda_{v_{n}}^{0} \rightarrow\left(P_{t}^{0, v}\right)^{*} \lambda_{v}^{0}=: \lambda_{v}(t)$, uniformly on $I$ as $n \rightarrow+\infty$. Hence it is not restrictive to assume that for every $n \in \mathbb{N}$ and $t \in I$,

$$
\lambda_{v_{n}}(t) \in\left(\Delta_{\gamma_{v_{n}}(t)}^{2}\right)^{\perp} \backslash\left(\Delta_{\gamma_{v_{n}}(t)}^{3}\right)^{\perp}
$$


Since the controls $v_{n}$ can be recovered by the formula ${ }^{7}$ (see, e.g., [21])

$$
\left(\begin{array}{c}
v_{n, 1}(t) \\
v_{n, 2}(t)
\end{array}\right)=\frac{1}{\left(h_{112}\left(\lambda_{v_{n}}(t)\right)^{2}+h_{212}\left(\lambda_{v_{n}}(t)\right)^{2}\right)^{1 / 2}}\left(\begin{array}{c}
-h_{212}\left(\lambda_{v_{n}}(t)\right) \\
h_{112}\left(\lambda_{v_{n}}(t)\right)
\end{array}\right)
$$

we argue that the convergence of $v_{n}$ to 0 holds in $L^{\infty}(I, \mathbb{R}) \oplus L^{2}(I, \mathbb{R})$ as well. Recalling the smooth immersion $\phi$ defined in (4.3), we finally conclude that

$$
\lim _{n \rightarrow \infty}\left(v_{n, 1}, \phi\left(v_{n, 2}\right)\right)=0
$$

in $L^{\infty}(I, R) \oplus \mathbb{R} \oplus L^{2}(I, R)$, whence we can assume without loss of generality to work within a neighborhood $\mathcal{W}$ where Theorem 1.3 applies.

We complete the proof by showing that all the singular controls $\left(v_{1}, v_{2}\right) \in \Omega(y)$ such that $\left(v_{1}, \phi\left(v_{2}\right)\right)$ is contained in $\mathcal{W}$ are of the form $\left(v_{1}, 0\right)$ with $\int_{0}^{1} v_{1}(t) \mathrm{d} t=0$. More precisely, we will show that every singular control $z=(v, c, w) \in \mathcal{W}$ is such that $c=w=0$.

Let $z=(v, c, w)$ be such a control. By Theorem 1.3 we readily deduce that $z \in \operatorname{ker}\left(d_{0} \stackrel{\circ}{F}\right)$, and that $\lambda \operatorname{He}_{0} \stackrel{\circ}{F}(z)=$ 0 .

It is also not difficult to compute that for every $z \in \operatorname{ker}\left(d_{0} \stackrel{\circ}{F}\right)$ and $x=\left(x^{\prime}, x^{\prime \prime}\right) \in \operatorname{ker}\left(d_{0} \stackrel{\circ}{F}\right) \oplus \stackrel{\circ}{E}$ we have

$$
d_{z} \stackrel{\circ}{F}(x)=\left(2 \lambda \mathrm{He}_{0} \stackrel{\circ}{F}\left(z, x^{\prime}\right), d_{0} \stackrel{\circ}{F}\left(x^{\prime \prime}\right)\right) .
$$

Thus for every $x \in \operatorname{ker}\left(d_{0} \stackrel{\circ}{F}\right)$ we deduce that $d_{z} \stackrel{\circ}{F}(x)=\left(2 \lambda \operatorname{He}_{0} \stackrel{\circ}{F}\left(z, x^{\prime}\right), 0\right)$. Let $0 \neq \xi \in \operatorname{Im}\left(d_{z} \stackrel{\circ}{F}\right)^{\perp}$; by continuity it is not restrictive to assume that the $\mathbb{R}$-component of $\xi$ is different from zero, i.e., $\xi_{1} \neq 0$.

By (5.14) we then argue that for every $x \in \operatorname{ker}\left(d_{0} \stackrel{\circ}{F}\right)$ we have:

$$
0=\xi d_{z} \stackrel{\circ}{F}(x)=2 \xi_{1} \lambda \mathrm{He}_{0} \stackrel{\circ}{F}(z, x),
$$

i.e, $z \in \operatorname{ker}\left(\lambda \mathrm{He}_{0} \stackrel{\circ}{F}\right)$. We conclude that $z=(v, 0,0)$, with $v$ a zero-mean control, since $\gamma$ is rank-two-nice by assumption.

\section{JACOBI FIELDS AND COMPUTATIONS OF CONJUGATE POINTS}

We explain in this last chapter how to characterize conjugate points along a rank-two-nice singular curve $\gamma$. We suppose for simplicity that $\Delta_{x}=\operatorname{span}\left\{X_{1}(x), X_{2}(x)\right\}$ in the domain under consideration and we consider, as in Definition 2.7, a local chart $\mathcal{V}_{1} \in L^{2}\left(I, \mathbb{R}^{2}\right)$ centered at zero.

For every $s \in I$ we define

$$
\mathcal{V}_{1}^{s}:=\left\{v \cdot \chi_{[0, s]} \mid v \in \mathcal{V}_{1}\right\} \subset L^{2}\left([0, s], \mathbb{R}^{2}\right)
$$

Similarly, if $\gamma$ is an admissible curve, $\gamma_{s}:=\left.\gamma\right|_{[0, s]}$ denotes the restriction of $\gamma$ to $[0, s]$. The endpoint map, the energy and the extended endpoint map have natural restrictions to $\mathcal{V}_{1}^{s}$, which we denote by $F_{s}, J_{s}$ and $\mathcal{F}_{s}$, respectively. In particular $F_{s}(\gamma)=F\left(\gamma_{s}\right), J_{s}(\gamma)=J\left(\gamma_{s}\right)$ and $\mathcal{F}_{s}(\gamma)=\mathcal{F}\left(\gamma_{s}\right)$.

Fix a rank-two-nice curve $\gamma$. To detect conjugate times it is convenient to work on the cotangent space $T^{*} M$. We normalize the extremal lift $t \mapsto \lambda(t)$ of $\gamma$ so that $\lambda_{0}:=\lambda(0) \in T_{x_{0}}^{*} M$ has norm one. Then for every $t \in[0, s]$

\footnotetext{
${ }^{7}$ For every $i, j, k \in\{1,2\}, \eta \in T^{*} M$, and for $\pi: T^{*} M \rightarrow M$ denoting the bundle projection, the notation $h_{i j k}(\eta)$ stands for the product $\left\langle\eta,\left[X_{i},\left[X_{j}, X_{k}\right]\right](\pi(\eta))\right\rangle$.
} 
we have:

$$
\lambda(t)=\left(e^{-t X_{1}}\right)^{*} \lambda_{0}=\left(e^{(s-t) X_{1}}\right)^{*} \lambda(s) .
$$

For future purposes we also define the vector field

$$
g_{t}^{s}:=e_{*}^{(s-t) X_{1}} X_{2}
$$

Notice that $g_{t}^{1}$ is the vector field $g_{t}$ introduced in the previous sections.

To characterize the set of conjugate times along $\gamma$ for the map $F$ (resp. for the extended endpoint map $\mathcal{F}$, i.e., for $F$ restricted onto the level sets of $J)$, we consider the sets $K_{1}^{s}, K_{2}^{s} \subset L^{\infty}([0, s], \mathbb{R}) \oplus \mathbb{R} \oplus L^{2}([0, s], \mathbb{R})$ :

$$
\begin{aligned}
& K_{1}^{s}=\left\{(v, c, w) \mid \int_{0}^{s} w(t) \dot{g}_{t}^{s}(\gamma(s)) \mathrm{d} t=\int_{0}^{s} v(t) \mathrm{d} t X_{1}(\gamma(s))+c X_{2}(\gamma(s))\right\}=\operatorname{ker}\left(d_{0} \stackrel{\circ}{F}_{s}\right), \\
& K_{2}^{s}=\left\{(v, c, w) \in K_{1}^{s} \mid \int_{0}^{s} v(t) \mathrm{d} t=0\right\},
\end{aligned}
$$

and we study on either one of these sets the quadratic form

$$
\int_{0}^{s}\left\langle\lambda(s),\left[\dot{g}_{t}^{s}, g_{t}^{s}\right](\gamma(s))\right\rangle w(t)^{2} \mathrm{~d} t+\int_{0}^{s}\left\langle\lambda(s),\left[c X_{2}+\int_{0}^{t} w(\tau) \dot{g}_{\tau}^{s} \mathrm{~d} \tau, w(t) \dot{g}_{t}^{s}\right](\gamma(s))\right\rangle \mathrm{d} t .
$$

\subsection{The symplectic framework}

Let $\sigma \in \Lambda^{1}\left(T^{*} M\right)$ be the standard Liouville one-form and let $\omega:=d \sigma \in \Lambda^{2}\left(T^{*} M\right)$ denote the canonical symplectic form on $T^{*} M$. We introduce the following notations:

- For every $\mu \in T^{*} M$ and $x \in T_{\mu}\left(T^{*} M\right)$, we denote by

$$
x^{<}:=\left\{y \in T_{\mu}\left(T^{*} M\right) \mid \omega_{\mu}(x, y)=0\right\}
$$

the skew-orthogonal complement to $x$ in $T_{\mu}\left(T^{*} M\right)$;

- For every subspace $N \subset T T^{*} M$, we denote by

$$
N^{<}:=\left\{y \in T T^{*} M \mid \omega(x, y)=0, \text { for every } x \in N\right\}
$$

the skew-orthogonal complement to $N$;

- For every Hamiltonian function $a \in C^{\infty}\left(T^{*} M\right)$, we denote by $\vec{a} \in \operatorname{Vec}\left(T^{*} M\right)$ its Hamiltonian lift, given by the formula

$$
\omega_{\mu}(\cdot, \vec{a})=d_{\mu} a(\cdot), \text { for every } \mu \in T^{*} M .
$$

Fix $s \in I$, and let $\pi: T^{*} M \rightarrow M$ be the canonical bundle projection. For every $t \in[0, s]$, we introduce the fiberwise linear Hamiltonians

$$
\begin{aligned}
\xi_{1}: T^{*} M & \rightarrow \mathbb{R}, & \eta_{t}^{s}: T^{*} M & \rightarrow \mathbb{R}, \\
\mu & \mapsto\left\langle\mu, X_{1}(\pi(\mu))\right\rangle, & \mu & \mapsto\left\langle\mu, g_{t}^{s}(\pi(\mu))\right\rangle .
\end{aligned}
$$

Accordingly, we define the Hamiltonian lifts $\vec{\xi}_{1}$ and $\vec{\eta}_{t}^{s}$, and we observe that $\vec{\eta}_{s}^{s}=\vec{\xi}_{2}$, where $\xi_{2}$ is the Hamiltonian function associated with $X_{2}$ as in (6.4). 
We identify $T_{\gamma(s)}^{*} M$ with $T_{\lambda(s)}\left(T_{\gamma(s)}^{*} M\right)$ : in terms of local bases we identify $\nu=\sum_{i=1}^{m} \nu_{i} \mathrm{~d} x_{i}$ and $\nu=$ $\sum_{i=1}^{m} \nu_{i} \partial_{\lambda_{i}}$. Then $\lambda(s)$ is associated with the value at $\lambda(s)$ of the Euler vector field $\mathbf{e} \in \operatorname{Vec}\left(T^{*} M\right)$ :

$$
\mathbf{e}(\lambda(s))=\lambda_{1}(s) \partial_{\lambda_{1}}+\cdots+\lambda_{m}(s) \partial_{\lambda_{m}}
$$

Finally, we define:

- $\Sigma$ to be the skew-orthogonal complement of $\lambda(s)$ in the symplectic space $T_{\lambda(s)}\left(T^{*} M\right)$, modded out by $\operatorname{span}\{\lambda(s)\}$. In formula:

$$
\Sigma:=\left\{\nu \in T_{\lambda(s)}\left(T^{*} M\right) \mid \omega_{\lambda(s)}(\lambda(s), \nu)=0\right\} / \lambda(s) .
$$

Notice that $\Sigma$ is a symplectic subspace of $T_{\lambda(s)}\left(T^{*} M\right)$, of dimension $2(m-1)$.

- $\Pi:=T_{\gamma(s)}^{*} M / \lambda(s)$. Then $\Pi$ is a Lagrangian (i.e. of dimension $\left.m-1\right)$ subspace of $\Sigma$, that can be identified with $T_{\lambda(s)}\left(T_{\gamma(s)}^{*} M\right) / \mathbf{e}(\lambda(s))$.

For every $\mu \in T_{\gamma(s)}^{*} M$ we have:

$$
\omega_{\lambda(s)}\left(\mu, \vec{\xi}_{1}\right):=\left\langle\mu, X_{1}(\gamma(s))\right\rangle, \omega_{\lambda(s)}\left(\mu, \vec{\eta}_{t}^{s}\right):=\left\langle\mu, g_{t}^{s}(\gamma(s))\right\rangle
$$

where $\langle\cdot, \cdot\rangle$ denotes the dual product. In particular $\omega_{\lambda(s)}\left(\lambda(s), \vec{\xi}_{1}\right)=\omega_{\lambda(s)}\left(\lambda(s), \vec{\eta}_{t}^{s}\right)=0$ for every $t \in[0, s]$, and thus both $\vec{\xi}_{1}$ and $\vec{\eta}_{t}^{s}$ can be identified with elements of $\Sigma$.

By the equality $\omega_{\lambda(s)}\left(\vec{\xi}_{1}, \vec{\eta}_{t}^{s}\right)=\left\langle\lambda(s),\left[X_{1}, g_{t}^{s}\right](\gamma(s))\right\rangle$, we deduce that $\omega_{\lambda(s)}\left(\vec{\xi}_{1}, \vec{\eta}_{t}^{s}\right) \equiv 0$ for every $t \in[0, s]$, whence

$$
\omega_{\lambda(s)}\left(\vec{\xi}_{1}, \dot{\vec{\eta}}_{t}^{s}\right) \equiv 0, \text { for every } t \in[0, s]
$$

Let $l_{t, s}^{0}:=\omega_{\lambda(s)}\left(\dot{\vec{\eta}}_{t}^{s}, \vec{\eta}_{t}^{s}\right)=\left\langle\lambda(s),\left[\dot{g}_{t}^{s}, g_{t}^{s}\right](\gamma(s))\right\rangle$. The quadratic form (6.2) becomes

$$
\int_{0}^{s} l_{t, s}^{0} w(t)^{2} \mathrm{~d} t+\int_{0}^{s} \omega_{\lambda(s)}\left(c \vec{\xi}_{2}+\int_{0}^{t} w(\tau) \dot{\vec{\eta}}_{\tau}^{s} \mathrm{~d} \tau, w(t) \dot{\vec{\eta}}_{t}^{s}\right) \mathrm{d} t
$$

while the symplectic counterparts of the domain conditions (6.1) read

$$
\begin{aligned}
K_{1}^{s, \sharp} & =\left\{(c, w) \in \mathbb{R} \oplus L^{2}([0, s], \mathbb{R}) \mid c \vec{\xi}_{2}(\lambda(s))+\int_{0}^{s} w(t) \dot{\vec{\eta}}_{t}^{s}(\lambda(s)) \mathrm{d} t \in \mathbb{R} \cdot \vec{\xi}_{1}(\lambda(s))+\Pi\right\}, \\
K_{2}^{s, \sharp} & =\left\{(c, w) \in \mathbb{R} \oplus L^{2}([0, s], \mathbb{R}) \mid c \vec{\xi}_{2}(\lambda(s))+\int_{0}^{s} w(t) \dot{\vec{\eta}}_{t}^{s}(\lambda(s)) \mathrm{d} t \in \Pi\right\} .
\end{aligned}
$$

Let $\pi_{L^{2}([0, s], \mathbb{R})}: \mathbb{R} \oplus L^{2}([0, s], \mathbb{R}) \rightarrow L^{2}([0, s], \mathbb{R})$ denote the standard projection. Then $w \in \pi_{L^{2}([0, s], \mathbb{R})}\left(K_{1}^{s, \sharp)}\right.$ if and only if

$$
\omega_{\lambda(s)}\left(\nu, \int_{0}^{s} \dot{\vec{\eta}}_{t}^{s} w(t) \mathrm{d} t\right)=0
$$


for every ${ }^{8} \nu \in \Pi \cap \operatorname{span}\left\{\vec{\xi}_{1}(\lambda(s)), \vec{\xi}_{2}(\lambda(s))\right\}^{<}+\mathbb{R} \cdot \vec{\xi}_{1}(\lambda(s))$. Likewise, $w \in \pi_{L^{2}([0, s], \mathbb{R})}\left(K_{2}^{s, \sharp}\right)$ if and only if

$$
\omega_{\lambda(s)}\left(\nu, \int_{0}^{s} \dot{\vec{\eta}}_{t}^{s} w(t) \mathrm{d} t\right)=0
$$

for every $\nu \in \Pi \cap \vec{\xi}_{2}(\lambda(s))^{<}$.

In particular, this argument shows that the subspace $\left\{w \mid w \in \pi_{L^{2}([0, s], \mathbb{R})}\left(K_{1}^{s, \sharp}\right)\right\}$ is the $L^{2}([0, s], \mathbb{R})$ orthogonal complement of the subspace

$$
\left\{\omega_{\lambda(s)}\left(\nu, \dot{\vec{\eta}}_{t}^{s}\right) \mid \nu \in \Pi \cap \operatorname{span}\left\{\vec{\xi}_{1}(\lambda(s)), \vec{\xi}_{2}(\lambda(s))\right\}^{<}+\mathbb{R} \cdot \vec{\xi}_{1}(\lambda(s)\}\right.
$$

similarly, we deduce that the subspace $\left\{w \mid w \in \pi_{L^{2}([0, s], \mathbb{R})}\left(K_{2}^{s, \sharp}\right)\right\}$ is the $L^{2}([0, s], \mathbb{R})$-orthogonal complement of the subspace

$$
\left\{\omega_{\lambda(s)}\left(\nu, \dot{\vec{\eta}}_{t}^{s}\right) \mid \nu \in \Pi \cap \vec{\xi}_{2}(\lambda(s))^{<}\right\}
$$

\subsection{Jacobi equations}

Let $s \in I$.

Definition 6.1. We say $\gamma(s)$ is a conjugate point along $\gamma$ if the Hessian map in (6.8), defined on the domain determined by either one of the two conditions in (6.9), has a kernel that strictly contains the subspace $\check{Z}_{1}$ of zero-mean controls. The multiplicity of $\gamma(s)$ as a conjugate point is the codimension of $\check{Z}_{1}$ in this kernel (compare with Def. 4.2).

Given $(c, w) \in K_{1}^{s, \sharp}\left(\right.$ resp. $\left.(c, w) \in K_{2}^{s, \sharp}\right)$, we define

$$
Y_{(c, w)}(t):=c \vec{\xi}_{2}(\lambda(s))+\int_{0}^{t} w(\tau) \dot{\vec{\eta}}_{\tau}^{s}(\lambda(s)) \mathrm{d} \tau
$$

Upon integrating by part we see that the bilinear form associated with (6.8) is given by (see also [6], Chap. 4):

$$
\int_{0}^{s} l_{t, s}^{0} w_{0}(t) w(t) \mathrm{d} t+\int_{0}^{s} \omega_{\lambda(s)}\left(Y_{\left(c_{0}, w_{0}\right)}(t), \dot{Y}_{(c, w)}(t)\right) \mathrm{d} t
$$

It follows from (6.9) that $\gamma(s)$ is a conjugate point along $\gamma$ if and only if there exists $\left(c_{0}, w_{0}\right) \in K_{1}^{s, \sharp}$ (resp. in $K_{2}^{s, \sharp)}$ such that

$$
\int_{0}^{s}\left(l_{t, s}^{0} w_{0}(t)+\omega_{\lambda(s)}\left(c_{0} \vec{\xi}_{2}+\int_{0}^{t} w_{0}(\tau) \dot{\vec{\eta}}_{\tau}^{s} \mathrm{~d} \tau, \dot{\vec{\eta}}_{t}^{s}\right)\right) w(t) \mathrm{d} t=0
$$

for every $w \in \pi_{L^{2}([0, s], \mathbb{R})}\left(K_{1}^{s, \sharp}\right)\left(\right.$ resp. $\left.w \in \pi_{L^{2}([0, s], \mathbb{R})}\left(K_{2}^{s, \sharp}\right)\right)$.

${ }^{8}$ Here $\nu$ can be defined modulo $\vec{\xi}_{1}(\lambda(s))$ because of (6.7). 
We conclude that there exists $\nu \in \Pi \cap \operatorname{span}\left\{\vec{\xi}_{1}(\lambda(s)), \vec{\xi}_{2}(\lambda(s))\right\}^{<}+\mathbb{R} \cdot \vec{\xi}_{1}(\lambda(s))\left(\right.$ resp. $\left.\nu \in \Pi \cap \vec{\xi}_{2}(\lambda(s))^{<}\right)$such that

$$
l_{t, s}^{0} w_{0}(t)+\omega_{\lambda(s)}\left(c_{0} \vec{\xi}_{2}+\int_{0}^{t} w_{0}(\tau) \dot{\vec{\eta}}_{\tau}^{s} \mathrm{~d} \tau, \dot{\vec{\eta}}_{t}^{s}\right)=-\omega_{\lambda(s)}\left(\nu, \dot{\vec{\eta}}_{t}^{s}\right), \quad \text { a.e. } t \in[0, s]
$$

By multiplying both its sides by $\dot{\vec{\eta}}_{t}^{s}(\lambda(s))$, we rewrite (6.15) as the Jacobi equation on $\Sigma$ :

$$
\left.l_{t, s}^{0} \dot{k}(t)=\omega_{\lambda(s)} \dot{\vec{\eta}}_{t}^{s}, k(t)\right) \dot{\vec{\eta}}_{t}^{s}(\lambda(s))
$$

where $k(t):=c_{0} \vec{\xi}_{2}(\lambda(s))+\int_{0}^{t} w_{0}(\tau) \dot{\vec{\eta}}_{\tau}^{s}(\lambda(s)) \mathrm{d} \tau+\nu$ and the boundary conditions are given by

$$
\begin{aligned}
& \text { (a) }\left\{\begin{array}{l}
k(0) \in \Pi \cap \operatorname{span}\left\{\vec{\xi}_{1}(\lambda(s)), \vec{\xi}_{2}(\lambda(s))\right\}^{<}+\operatorname{span}\left\{\vec{\xi}_{1}(\lambda(s)), \vec{\xi}_{2}(\lambda(s))\right\} \\
k(s) \in \Pi
\end{array}\right. \\
& \text { (b) }\left\{\begin{array}{l}
k(0) \in \Pi \cap \operatorname{span}\left\{\vec{\xi}_{2}(\lambda(s))\right\}^{<}+\operatorname{span}\left\{\vec{\xi}_{2}(\lambda(s))\right\} \\
k(s) \in \Pi
\end{array}\right.
\end{aligned}
$$

Proposition 6.2. Let $s \in I$. The point $\gamma(s)$ is a conjugate point along $\gamma$ for the map $F_{s}$ (resp. for $\mathcal{F}_{s}$ ) if and only if there exists a solution $k(\cdot)$ to (6.16) that satisfies the boundary conditions (6.17).

Remark 6.3. Observe that in both cases $k(0)$ belongs to a Lagrangian subspace of $\Sigma$, of the form $\Pi \cap \Gamma^{<}+\Gamma$ with $\Gamma$ isotropic.

\subsection{Regular distributions}

We specify the Jacobi equations for a particular class of rank-two sub-Riemannian structures, the so-called regular structures, that have been investigated e.g. in [14, 21]. Assume $M$ is an $(m+2)$-dimensional manifold, and that $\Delta=\operatorname{span}\left\{X_{1}, X_{2}\right\}$ in the domain under consideration. Moreover, we assume that:

- $X_{1}, X_{2}, \ldots,\left(\operatorname{ad} X_{1}\right)^{m-1} X_{2}$ are linearly independent.

- There exist smooth functions $\beta,\left\{\alpha^{i}, i=0, \ldots, m-1\right\}$ on $M$, such that

$$
\left(\operatorname{ad} X_{1}\right)^{m} X_{2}=\beta X_{1}+\sum_{i=0}^{m-1} \alpha^{i}\left(\operatorname{ad} X_{1}\right)^{i} X_{2}
$$

- $\left[\left[X_{1}, X_{2}\right], X_{2}\right]$ is linearly independent from $V=\operatorname{span}\left\{X_{1}, X_{2}, \ldots,\left(\operatorname{ad} X_{1}\right)^{m-1} X_{2}\right\}$.

Under these hypotheses it turns out ([21], Sect. 8) that integral curves of the vector field $X_{1}$ are indeed corankone abnormal geodesics for $\Delta$. Moreover, these curves are also strictly abnormal as soon as $\beta \neq 0$ along the trajectory.

With $g_{t}^{s}$ as in (6.4) and $i \in \mathbb{N}$, we define

$$
\begin{aligned}
g_{t}^{s,(i)} & :=\partial_{t}^{(i)} g_{t}^{s}=(-1)^{i} e_{*}^{(s-t) X_{1}}\left(\operatorname{ad} X_{1}\right)^{i} X_{2}, \\
l_{t, s}^{(i)} & :=\left\langle\lambda(s),\left[g_{t}^{s,(1)}, g_{t}^{s,(i)}\right](\gamma(s))\right\rangle=\omega_{\lambda(s)}\left(\vec{\eta}_{t}^{s,(1)}, \vec{\eta}_{t}^{s,(i)}\right) .
\end{aligned}
$$


Calling $\beta_{t}:=\beta(\gamma(t))$ and $\alpha_{t}^{i}:=\alpha^{i}(\gamma(t))$, it is immediate to deduce from (6.18) its symplectic version along $\gamma$, that is

$$
\vec{\eta}_{t}^{s,(m)}(\lambda(s))=\beta_{t} \vec{\xi}_{1}(\lambda(s))+\sum_{i=0}^{m-1} \alpha_{t}^{i} \vec{\eta}_{t}^{s,(i)}(\lambda(s))
$$

Let $Z:=\left\{\vec{\xi}_{1}(\lambda(s)), \vec{\eta}_{t}^{s}(\lambda(s)), t \in[0, s]\right\}$, and notice that we have the decomposition $\Sigma=\Pi \oplus Z$. For every $\tau \in[0, s],(6.20)$, yields:

$$
Z=\operatorname{span}\left\{\vec{\xi}_{1}(\lambda(s)), \vec{\eta}_{\tau}^{s}(\lambda(s)), \ldots, \vec{\eta}_{\tau}^{s,(m-1)}(\lambda(s))\right\}
$$

Notice that $Z$ is not a Lagrangian subspace, nonetheless the symplectic form $\omega_{\lambda(s)}$ defines a non-degenerate splitting between $\Pi$ and $Z$.

Let us write $k(t)=z_{t}+\theta_{t}$, with $z_{t} \in Z$ and $\theta_{t} \in \Pi$. Then (6.16) splits as the differential system of equations:

$$
\left\{\begin{aligned}
l_{t, s}^{0} \dot{z}_{t} & =\omega_{\lambda(s)}\left(\vec{\eta}_{t}^{s,(1)}, z_{t}+\theta_{t}\right) \vec{\eta}_{t}^{s,(1)}(\lambda(s)), \\
\dot{\theta}_{t} & =0
\end{aligned}\right.
$$

whose boundary conditions are given respectively by (compare with (6.17)):

$$
\begin{array}{lll}
\text { (a) } \quad z_{s}=0, & z_{0} \in \operatorname{span}\left\{\vec{\xi}_{1}(\lambda(s)), \vec{\xi}_{2}(\lambda(s))\right\}, & \omega_{\lambda(s)}\left(\vec{\xi}_{2}, \theta_{0}\right)=\omega_{\lambda(s)}\left(\vec{\xi}_{1}, \theta_{0}\right)=0, \\
\text { (b) } \quad z_{s}=0, \quad z_{0} \in \operatorname{span}\left\{\vec{\xi}_{2}(\lambda(s))\right\}, & \omega_{\lambda(s)}\left(\vec{\xi}_{2}, \theta_{0}\right)=0 .
\end{array}
$$

If we write $z_{t}=z_{t}^{f} \vec{\xi}_{1}(\lambda(s))+\sum_{i=0}^{m-1} z_{t}^{i} \vec{\eta}_{t}^{s,(i)}(\lambda(s))$ and we define $\zeta_{t}:=\omega_{\lambda(s)}\left(\vec{\eta}_{t}^{s}, \theta_{0}\right)$ and $l_{t, s}^{(i)}$ as in (6.19), we see that (6.22) is equivalent to the following system of equations:

$$
\left\{\begin{array}{rlrl}
\dot{z}_{t}^{f} & =-\beta_{t} z_{t}^{m-1}, & z_{s}^{f}=0, \\
\dot{z}_{t}^{0} & =-\alpha_{t}^{0} z_{t}^{m-1}, & z_{s}^{0}=0, \\
l_{t, s}^{0}\left(\dot{z}_{t}^{1}+\alpha_{t}^{1} z_{t}^{m-1}\right) & =\sum_{j=2}^{m-1} l_{t, s}^{(j)} z_{t}^{j}+\dot{\zeta}_{t}, & z_{s}^{1}=0, \\
\dot{z}_{t}^{j}+\alpha_{t}^{j} z_{t}^{m-1} & =-z_{t}^{j-1}, \text { for every } j=2, \ldots, m-1, & z_{s}^{j}=0, \\
\zeta_{t}^{(m)} & =\beta_{t} \omega_{\lambda(s)}\left(\vec{\xi}_{1}, \theta_{0}\right)+\sum_{i=0}^{m-1} \alpha_{t}^{i} \zeta_{t}^{(i)}, & \zeta_{s} & =0,
\end{array}\right.
$$

and $\gamma(s)$ is a conjugate point along $\gamma$ for $F_{s}$ (resp. for $\mathcal{F}_{s}$ ) if and only if (6.24) admits a nontrivial solution that further satisfies the boundary conditions (6.23)

(a) $z_{0}^{i}=0$ for all $i=1, \ldots, m-1$,

(b) $z_{0}^{i}=0$ for all $i=1, \ldots, m-1$ and also $z_{0}^{f}=0$.

\subsection{The Engel case}

We study conjugate points along rank-two-nice singular curves on Engel structures, that is regular rank-two sub-Riemannian structures $(M, \Delta)$ with $M$ of dimension 4 . In this case, the vector field $X_{1}$ satisfies all the conditions on the Lie brackets required in the previous part by the results in [10]. 
We begin with the endpoint map $F_{s}$. Taking into account that $\omega_{\lambda(s)}\left(\vec{\xi}_{1}, \theta_{0}\right)=0$, the relevant equations to solve (6.24) are:

$$
\left\{\begin{aligned}
l_{t, s}^{0}\left(\dot{z}_{t}^{1}+\alpha_{t}^{1} z_{t}^{1}\right) & =\dot{\zeta}_{t}, & & z_{s}^{1}=0 \\
\ddot{\zeta}_{t} & =\alpha_{t}^{0} \zeta_{t}+\alpha_{t}^{1} \dot{\zeta}_{t}, & \zeta_{s} & =0 .
\end{aligned}\right.
$$

We see from (6.20) that

$$
i_{t, s}^{0}=\frac{\mathrm{d}}{\mathrm{d} t} \omega_{\lambda(s)}\left(\dot{\vec{\eta}}_{t}^{s}, \vec{\eta}_{t}^{s}\right)=\omega_{\lambda(s)}\left(\ddot{\vec{\eta}}_{t}^{s}, \vec{\eta}_{t}^{s}\right)=\alpha_{t}^{1} l_{t, s}^{0}
$$

yielding that $l_{t, s}^{0}=l_{0, s}^{0} e^{\int_{0}^{t} \alpha_{\tau}^{1} \mathrm{~d} \tau}$, and therefore

$$
l_{0, s}^{s} \frac{\mathrm{d}}{\mathrm{d} t}\left(z_{t}^{1} e^{-\int_{0}^{t} \alpha_{\tau}^{1} \mathrm{~d} \tau}\right)=\dot{\zeta}_{t}
$$

Because $\gamma$ is rank-two-nice, $l_{0, s}^{0} \neq 0$ and then the further requirement $z_{0}^{1}=0$ is met if and only if $\zeta_{0}=$ $\omega_{\lambda(s)}\left(\vec{\eta}_{0}^{s}, \theta_{0}\right)=0$. So far, we have thus established the relations

$$
\omega_{\lambda(s)}\left(\vec{\eta}_{s}^{s}, \theta_{0}\right)=\omega_{\lambda(s)}\left(\vec{\eta}_{0}^{s}, \theta_{0}\right)=\omega_{\lambda(s)}\left(\vec{\xi}_{1}, \theta_{0}\right)=0 .
$$

On the other hand $\theta_{0} \in \Pi$ belongs to a three dimensional space, and then the linear map $\omega_{\lambda(s)}\left(\cdot, \theta_{0}\right)$ cannot have a three dimensional kernel, for otherwise $\theta_{0}$ would be zero. This implies that $\gamma(s) \in I$ is a conjugate point along $\gamma$ if and only if

$$
X_{1}(\gamma(s)) \wedge X_{2}(\gamma(s)) \wedge g_{0}^{s}(\gamma(s))=0 .
$$

Similar computations hold for the extended endpoint map $\mathcal{F}_{s}$. Here, the relevant equations are

$$
\left\{\begin{aligned}
\dot{z}_{t}^{f} & =-\beta_{t} z_{t}^{1}, & z_{s}^{f} & =0, \\
l_{t, s}^{0}\left(\dot{z}_{t}^{1}+\alpha_{t}^{1} z_{t}^{1}\right) & =\dot{\zeta}_{t}, & & z_{s}^{1}=0 \\
\ddot{\zeta}_{t} & =\alpha_{t}^{0} \zeta_{t}+\alpha_{t}^{1} \dot{\zeta}_{t}+\beta_{t} \omega_{\lambda(s)}\left(\vec{\xi}_{1}, \theta_{0}\right), & \zeta_{s} & =0 .
\end{aligned}\right.
$$

In addition to the equations found before, we require $z_{0}^{f}=0$. Since $z_{0}^{1}=\zeta_{0}$, we obtain from (6.27) that $z_{t}^{1}=\frac{1}{l_{0, s}^{0}} \zeta_{t} e^{-\int_{0}^{t} \alpha_{\tau}^{1} \mathrm{~d} \tau}$, which readily yields $\int_{0}^{s} \beta_{t} \zeta_{t} e^{-\int_{0}^{t} \alpha_{r}^{1} \mathrm{~d} r} \mathrm{~d} t=0$.

In terms of vector fields we conclude that $\gamma(s)$ is a conjugate time along $\gamma$ if and only if

$$
X_{2}(\gamma(s)) \wedge g_{0}^{s}(\gamma(s)) \wedge \int_{0}^{s} \beta_{t} e^{-\int_{0}^{t} \alpha_{\tau}^{1} \mathrm{~d} \tau} g_{t}^{s}(\gamma(s)) \mathrm{d} t=0
$$

We are ready to complete the discussion of the example of Section 1.4. From the structural equations we see that

$$
\left[X_{1},\left[X_{1}, X_{2}\right]\right]=\frac{X_{1}}{2}-X_{2}
$$

whence $\alpha_{t}^{0} \equiv-1, \alpha_{t}^{1} \equiv 0$ and $\beta_{t} \equiv 1 / 2$. Moreover it is not restrictive to assume by (6.26) that $l_{t, s}^{0} \equiv 1$, and we suppose as well $\dot{\zeta}_{0}=1$. 
In the case of the endpoint map $F_{s}$ it is immediate to solve for $\zeta$, and we find $\zeta_{t}=\sin (t)$. A time $s \in I$ yields a conjugate point $\gamma(s)$ along $\gamma$ if and only if $\zeta_{s}=0$, whence the computation of $a_{F}$ readily follows.

We pass to $\mathcal{F}_{s}$, and we denote for simplicity $\ell:=\omega_{\lambda(s)}\left(\vec{\xi}_{1}, \theta_{0}\right) \neq 0$. Solving for $\zeta_{t}$ and $z_{t}^{f}$ (imposing $z_{0}^{f}=0$ ) yields:

$$
\begin{aligned}
\zeta_{t} & =\frac{1}{2}(\ell-\ell \cos (t)+2 \sin (t)), \\
z_{t}^{f} & =\int_{0}^{t} \frac{1}{2} \zeta_{\tau} \mathrm{d} \tau=\frac{1}{4}(2+\ell t-2 \cos (t)-\ell \sin (t)) .
\end{aligned}
$$

We look for solutions to $\zeta_{s}=z_{s}^{f}=0$. Clearing out $\ell$, we see that $\gamma(s)$ is a conjugate point if and only if $s$ solves

$$
\frac{2 \cos (s)-2+s \sin (s)}{2 \cos (s)-2}=0
$$

Now, notice that if $\cos (s)=1, z_{s}^{f}=0$ if and only if $s=0$ or $\ell=0$, which is not possible by assumption. Then we may forget about the denominator and arrive to the claimed expression for $a_{\mathcal{F}}$.

\section{REFERENCES}

[1] A.A. Agracev and R.V. Gamkrelidze, Exponential representation of flows and a chronological enumeration. Mat. Sb. (N.S.) 107 (1978) 467-532, 639.

[2] A. Agrachev, D. Barilari and U. Boscain, A Comprehensive Introduction to Sub-Riemannian Geometry. Cambridge Studies in Advanced Mathematics. Cambridge University Press (2019).

[3] A.A. Agrachev, Some open problems. In Geometric control theory and sub-Riemannian geometry. Vol. 5 of Springer INdAM Ser.. Springer, Cham (2014) 1-13.

[4] A.A. Agrachev, F. Boarotto and A. Lerario, Homotopically invisible singular curves. Calc. Var. Partial Differ. Equ. 56 (2017) 105.

[5] A.A. Agrachev and Y.L. Sachkov, Control theory from the geometric viewpoint. Control Theory and Optimization, II. Vol. 87 of Encyclopaedia of Mathematical Sciences. Springer-Verlag, Berlin (2004).

[6] A.A. Agrachev and A.V. Sarychev, Strong minimality of abnormal geodesics for 2-distributions. J. Dyn. Control Syst. 1 (1995) 139-176.

[7] A.A. Agrachev and A.V. Sarychev, Abnormal sub-Riemannian geodesics: Morse index and rigidity. Ann. Inst. Henri Poincaré Anal. Non Linéaire 13 (1996) 635-690.

[8] F. Boarotto and A. Lerario, Homotopy properties of horizontal path spaces and a theorem of Serre in subriemannian geometry. Commun. Anal. Geom. 25 (2017) 269-301.

[9] Y. Chitour, F. Jean and E. Trélat, Genericity results for singular curves. J. Differ. Geom. 73 (2006) $45-73$.

[10] V. Gershkovich, On simplest Engel structures on 4-manifolds. In Dynamical systems and applications. Vol. 4 of World Sci. Ser. Appl. Anal. World Sci. Publ., River Edge, NJ (1995) 279-294.

[11] M.R. Hestenes, Applications of the theory of quadratic forms in Hilbert space to the calculus of variations. Pacific J. Math. 1 (1951) 525-581.

[12] F. Hirsch and G. Lacombe, Elements of functional analysis. Translated from the 1997 French original by Silvio Levy. Vol. 192 of Graduate Texts in Mathematics. Springer-Verlag, New York (1999).

[13] L. Hsu, Calculus of variations via the Griffiths formalism. J. Differ. Geom. 36 (1992) 551-589.

[14] W. Liu and H.J. Sussmann, Shortest paths for sub-Riemannian metrics on rank-two distributions. Mem. Am. Math. Soc. 118 (1995).

[15] R. Montgomery, Abnormal minimizers. SIAM J. Control Optim. 32 (1994) 1605-1620.

[16] R. Montgomery, A tour of subriemannian geometries, their geodesics and applications. Vol. 91 of Mathematical Surveys and Monographs. American Mathematical Society, Providence, RI (2002).

[17] J. Moser, On the volume elements on a manifold. Trans. Am. Math. Soc. 120 (1965) 286-294.

[18] L.S. Pontryagin, V.G. Boltyanskii, R.V. Gamkrelidze and E.F. Mishchenko, The mathematical theory of optimal processes. Translated by D. E. Brown. A Pergamon Press Book. The Macmillan Co., New York (1964).

[19] L. Rifford, Sub-Riemannian geometry and optimal transport. Springer Briefs in Mathematics. Springer, Cham (2014).

[20] A.V. Saryčev, Index of second variation of a control system. Mat. Sb. (N.S.) 113 (1980) 464-486.

[21] H.J. Sussmann, A cornucopia of four-dimensional abnormal sub-Riemannian minimizers. In Sub-Riemannian geometry. Vol. 144 of Progr. Math. Birkhäuser, Basel (1996) 341-364.

[22] E. Trélat, Some properties of the value function and its level sets for affine control systems with quadratic cost. J. Dyn. Control Syst. 6 (2000) 511-541. 